\title{
On the beneficial effect of Noise in Vertex Localization
}

\author{
Konstantinos A. Raftopoulos \\ Stefanos D. Kollias \\ Dionyssios D. Sourlas \\ Marin Ferecatu
}

\begin{abstract}
A theoretical and experimental analysis related to the effect of noise in the task of vertex identification in unknown shapes is presented. Shapes are seen as real functions of their closed boundary. An alternative global perspective of curvature is examined providing insight into the process of noiseenabled vertex localization. The analysis reveals that noise facilitates in the localization of certain vertices. The concept of noising is thus considered and a relevant global method for localizing Global Vertices is investigated in relation to local methods under the presence of increasing noise. Theoretical analysis reveals that induced noise can indeed help localizing certain vertices if combined with global descriptors. Experiments with noise and a comparison to localized methods validate the theoretical results.
\end{abstract}

Keywords Noising · Global Vertices · Global Curvature · Shape Representation · Object Recognition · Shape Modeling · Incremental Noising · Vertex Localization

Konstantinos A. Raftopoulos, Dionyssios D. Sourlas IVML-NTUA, Iroon Polytexneiou 9, 15780, Athens

Tel.: +30-210-7722521

Fax: $+30-210-7722492$

E-mail: raftop@image.ntua.gr

Stefanos D. Kollias

University of Lincoln, Brayford Pool, Lincoln, Lincolnshire, LN6 7TS, UK

Tel: +4401522 837368

E-mail: skollias@lincoln.ac.uk

Marin Ferecatu

CEDRIC-CNAM 292 Rue St Martin FR-75141 Paris Cedex 03

Tel: +330140272296

Fax: +330140272296

E-mail: marin.ferecatu@cnam.fr 


\section{Introduction}

Curvature is a local descriptor. At a given point on a curve, it is defined as the rate of change of the tangent to the curve at this point. A vertex is defined through curvature, as a point where curvature attains global minimum or global maximum. A vertex on a smooth curve can be seen as the generalization of a corner on a polyline. Curvature, as a descriptor of shape (e.g. describing the boundary of planar shapes) possesses a rare combination of good properties: It is intrinsic, intuitive, concise, well defined, analytic, extensively studied, well understood and of an undisputed perceptual importance. However, there are two serious problems concerning its such use. One has to do with noise. In a noisy curve, having, that is, high frequency Fourier components (hfFc) of no perceptual importance, the local nature of curvature restricts it in describing the noise itself rather than the underlying shape. Knowing whether hfFc of a curve represent noise or not, would require solving the harder problem of recognizing the object. Since hfFc might be defining for certain shapes or just noise in others, their presence in unrecognized (unknown) shapes is considered problematic, albeit they may present useful shape information. In practice, they are usually eliminated from the boundary of all shapes, by means of a blind step of smoothing, at the risk of losing useful discriminating shape information. Smoothing also distorts the shape's metrics in an unpredictable manner, a highly undesirable effect whenever certain morphometric measurements are defining for classification. Another problem in relation to curvature as a descriptor, has to do with meaningfulness. Even in noise free curves, the local nature of curvature doesn't permit any kind of context by means of which one could differentiate between points of similar curvature with respect to their perceptual characteristics on different parts of the curve. The reason behind both of these problems is curvature's local nature. Any solution would have to defy the very definition of curvature.

The local nature of curvature is challenged in this manuscript. An attempt to address the above problems is presented, based on a global definition of curvature that permits noise invariance and differentiation based on location perceptual characteristics. The global definition of curvature presented herein is based on the theoretical findings of [1]. Curvature at a point is seen in relation to the rate of change (per infinitesimal arc length) of the point's distance, to the rest of the curve. It is thus distance based and location dependent, a fact that is further investigated in this manuscript. This global definition of curvature is shown to absorb noise and convey context in a way that noise becomes an enabler for vertex identification. The new concept of noising (as opposed to smoothing) is thus conceived and a new method for identifying vertices without even having to calculate curvature is presented. Experiments with induced noise validate the theoretical results. 


\section{Related Work}

Shape descriptors have been used for pattern recognition in image processing applications for many decades now [2]. Indeed, shape is a fundamental characteristic of objects in images, widely used in object matching, identification and classification tasks. Shape descriptors divide roughly between area based methods, which use interior shape points, and boundary based methods, which use boundary information only [3]. Area based descriptions (such as geometric moments, moments invariants $[4,5]$ and shape orientation [6], elongation [7] and circularity [8]) are less accurate and thus easier to compute and can be applied to data coming from low quality sensors. On the other hand, boundary based methods (such as Curvature Scale Space [9], Fourier descriptors [10] and shape contexts [11]) are more suitable for high precision computer vision tasks and need data of good quality, data which starts to become available today, due to recent progress in sensor technology [12].

The method presented in this work relates closer to the last category: the general approach is to describe shapes by real functions [13] with the goal of estimating local shape features (e.g. curvature estimation, corner detection). However, numerical differentiation is sensitive to noise, thus much effort in recent years focused in finding methods that are robust to noise. In this case, one either use a smoothing pre-processing step (which usually affects the shape in a non-predictable way [14]), or use approximation of the real data with smooth curves $[15,16]$. Due to the local nature of noise, global methods usually integrate over larger scale characteristics of the data, in an attempt to inject invariance into the algorithms [17]. Some of these approaches include geometric flows [18], integral invariants defined via distance functions [19], tensor voting [17], active shape models using prior distributions to support large deformations in diffeomorphic metric mappings [20] and arc detection based on arithmetic discrete lines [17]. For the integral invariant estimators, the authors of [21] obtain convergence results when the grid resolution tends to zero and provide explicit formulas for the kernel size, which guarantees uniform convergence for smooth enough curves.

A multi-scale corner detector has been proposed by the authors of [22]. It first uses an adaptive local curvature threshold instead of a single global threshold as the method in CSS [10] does and then the angles of corner candidates are checked in a dynamic region of support for eliminating falsely detected corners. In [23], a curvature estimator is proposed that considers all the possible shapes and selects the most probable one with a global optimization approach. The estimator uses local bounds on tangent directions defined by the maximal straight segments. The estimator is adapted to noisy contours by replacing maximal segments with maximal blurred digital straight segments. The authors of [24], propose a method for dominant point detection and polygonal representation of noisy and possibly disconnected curves based on the decomposition of the curve into a sequence of maximal blurred segments. 
Unlike the methods described above, for which noise has a detrimental effect and thus must be resisted, bypassed or eliminated, the approach presented herein improves on this point by transforming noise into a facilitating factor for vertex identification.

\section{Contribution}

This paper builds on the preliminary results of [25] extending its experimental findings but it also serves as the theoretical foundation of [25]. In particular, Global Vertices introduced here for the first time, engage global shape information in the inherently local task of identifying vertices. Due to their location at the extremes of the boundary, Global Vertices are considered undisputed points of extreme curvature exposing this way a hidden relationship between location and curvature. The present paper builds on the results of [1] and [25] adding the following elements:

- The concept of Global Vertices and the theoretical foundation of their existence.

- A formal mathematical analysis on noising and its relation to Global Vertices.

- New experiments with noising for localizing Global Vertices and the such comparison to other methods in these tasks.

- The concept of noising is evolved to that of incremental noising and its behavior is investigated in relation to vertex localization against various baseline methods at different degrees of locality and smoothing characteristics. The extension from noising to incremental noising introduces novel concepts that are related to the space filling properties of a curve, enabling further insight into the general concept of noising.

- A hidden link is discovered between the new concept of incremental noising and the one of progressive smoothing. This link is significant because two conceptually orthogonal methods are now for the first time connected, elucidating this way a unification playground by means of the space filling properties of curves.

The rest of the paper is structured as follows: Previous necessary material briefly revisited in section 4, the Algebraic Interaction of Localities and a Zero Crossing Analysis of the second GL equation are presented in sections 5 and 6 respectively. The method of Noising and its use in Global Vertex Localization are presented in section 7 while Experimental Evaluation is performed in sections 8 and 9 . The conclusion in section 10 closes the paper.

\section{Connection to Previous Material: The VAR descriptor and the Global-Local Equations}

For every point on a curve one may consider the sum of its distances to all the other points. In the discrete case this is a summation. In the continuous 
case it is an integral. This total distance of a point to all the other points (or to the rest of the curve as one may choose to see it) captures a relationship between location and curvature and this is important because location, as opposed to curvature, is not affected by noise. The VAR descriptor introduced in [1] is the above normalized total distance and the Global Local (GL) Equations (Eq. (7),(8),(9)) are based on VAR to describe relations between location and curvature on the curve. We briefly now introduce the reader to the essentials on VAR and the GL equations. To simplify the discourse all curves are assumed with the necessary continuity, differentiability and regularity assumptions, details on these can be found in [1]. These assumptions are necessary because differential geometry will be used and the mathematics have to make sense. However, these assumptions don't restrict in any way the usual reality about digital curves. Any continuous digital curve (polyline) can acquire these properties after a suitable treatment (smoothing) at its corners, at an infinitesimal level, that doesn't change the discrete appearance of the curve. In Fig. (1) various basic shapes, with corners or/and vertices (generalization of a corner) are shown together with their VAR descriptors. Even though VAR describes the location of the points on the curve, it is obvious that VAR's local extrema are related to corners/vertices in a global way that is not affected by noise. The resistance to noise has been investigated in [1] with various experiments. In [25] things advanced one step further showing that noise actually helps this representation of vertices, a concept that will be further analyzed in this manuscript. We proceed formally now introducing the necessary mathematical concepts that will be used for further analysis. Let $(0, \lambda] \subset \mathbb{R}$ and $\boldsymbol{\alpha}:(0, \lambda] \rightarrow \mathbb{R}^{2}$ a continuous one to one (equivalent to non self intersecting), planar curve of non zero length $\lambda$ in $\mathbb{R}^{2}$, parametrized with respect to the arc length $s$ with all the necessary assumptions.

Let us consider $\mathbb{R}^{2}$ endowed with the usual metric, the plane distance, derived by the usual norm $\|\cdot\|$ and the trajectory of $\boldsymbol{\alpha}$ as $\boldsymbol{\alpha}((0, \lambda])$ (the image of the interval $(0, \lambda]$ through the vector function $\boldsymbol{\alpha})$.

Definition 1 Let $\boldsymbol{x}_{\mathbf{0}}=\boldsymbol{\alpha}\left(s_{0}\right)$ be a point of $\boldsymbol{\alpha}$ and $\Delta$ the diameter ${ }^{1}$ of $\boldsymbol{\alpha}$. The function:

$$
v_{s_{0}}:(0, \lambda] \rightarrow(0, \Delta], s \mapsto v_{s_{0}}(s):=\left\|\boldsymbol{x}_{\mathbf{0}}-\boldsymbol{\alpha}(s)\right\|
$$

we call the view of $\boldsymbol{\alpha}$ from the point $\boldsymbol{x}_{\mathbf{0}} \equiv \boldsymbol{\alpha}\left(s_{0}\right)$.

The view function $v_{s_{0}}$ is well defined given a choice of $\boldsymbol{x}_{\mathbf{0}}$, it is also continuous as a norm in $\mathbb{R}^{2}$. The view functions map the arc length to the chord length of the curve. Lets denote with $s_{*}$ a certain value of the arc length parameter corresponding to a point on the curve where the unit tangent and unit normal vectors are now considered. $\dot{\boldsymbol{\alpha}}\left(s_{*}\right)=\boldsymbol{t}$ (the dot denotes the derivative with

\footnotetext{
1 Supremum of all pair-wise distances between points of $\boldsymbol{\alpha}$.
} 


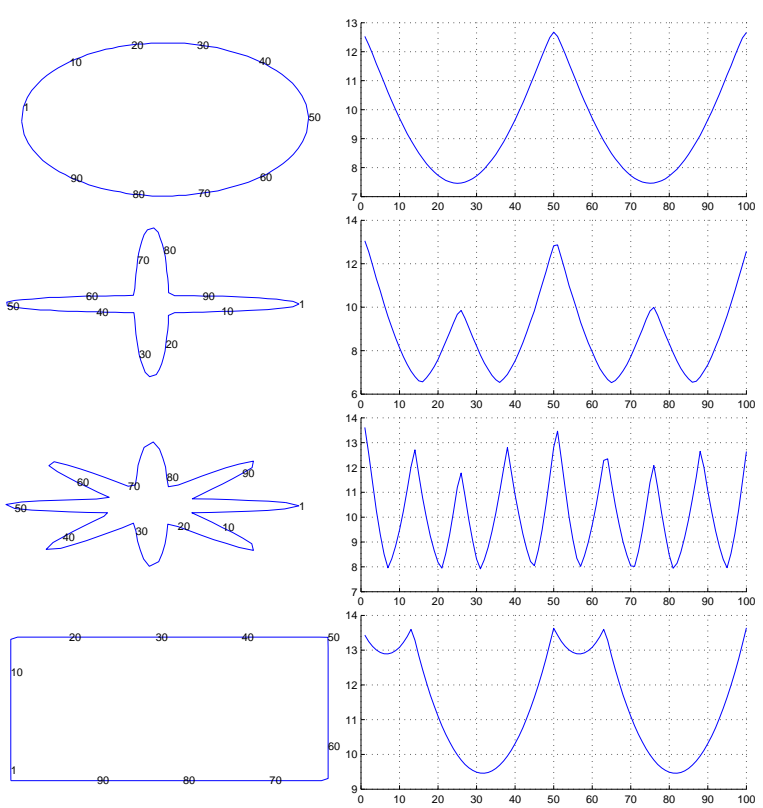

Fig. 1: Basic shapes (left column) and the corresponding View Area Representations (VARs, right column).

respect to the arc length $s)$ is the unit vector, tangent to the curve at $\boldsymbol{\alpha}\left(s_{*}\right)$ and $\boldsymbol{n}$ is the normal inward ${ }^{2}$ unit vector also at $\boldsymbol{\alpha}\left(s_{*}\right)$, with

$$
\frac{d \boldsymbol{t}}{d s}=-\kappa\left(s_{*}\right) \boldsymbol{n}, \frac{d \boldsymbol{n}}{d s}=\kappa\left(s_{*}\right) \boldsymbol{t}
$$

the Frenet equations for curve $\boldsymbol{\alpha}$ at $\boldsymbol{p}=\boldsymbol{\alpha}\left(s_{*}\right)$, with $\kappa\left(s_{*}\right)$ being the curvature at $\boldsymbol{p}$, as shown in Fig. 2. For the curvature $\kappa\left(s_{*}\right)$, we have adopted the sign convention, according to which, $\kappa(s)$ is positive when $\boldsymbol{n}(s)$ points away from the center of curvature. We also consider any choice of $\xi \in(0, \lambda], \xi \neq s_{*}$ and the respective point $\boldsymbol{q}=\boldsymbol{\alpha}(\xi)$. We call $\boldsymbol{r}\left(s_{*}, \xi\right)$ the vector $\boldsymbol{\alpha}\left(s_{*}\right)-\boldsymbol{\alpha}(\xi)$ and $\omega\left(s_{*}, \xi\right)$ the angle from $\boldsymbol{n}\left(s_{*}\right)$ to $-\boldsymbol{r}\left(s_{*}, \xi\right)$, measured counter-clockwise. Angle $\omega$ is a scalar function of two variables, $s$ and $\xi$; and $\boldsymbol{r}$ is a vector function of the same two variables; in both cases the variables will be omitted in the notation from now on.

Proposition 1 Let $\boldsymbol{\alpha}(s):(0, \lambda]) \rightarrow \mathbb{R}^{2}$ a closed, planar curve of nonzero length $\lambda$, as above. Let $\boldsymbol{p}=\boldsymbol{\alpha}\left(s_{*}\right)$ a point on the curve and $\boldsymbol{q}=\boldsymbol{\alpha}(\xi), \xi \neq s_{*}$ another point on the curve with $v_{\xi}(s)$ the respective view function. Then:

$$
\left.\frac{d}{d s} v_{\xi}\left(s_{*}\right) \equiv \frac{d}{d s} v_{\xi}(s)\right|_{s=s_{*}}=-\left.\sin (\omega)\right|_{s=s_{*}}
$$

\footnotetext{
${ }^{2}$ Directed to the interior of the bounded region defined by $\boldsymbol{\alpha}$.
} 


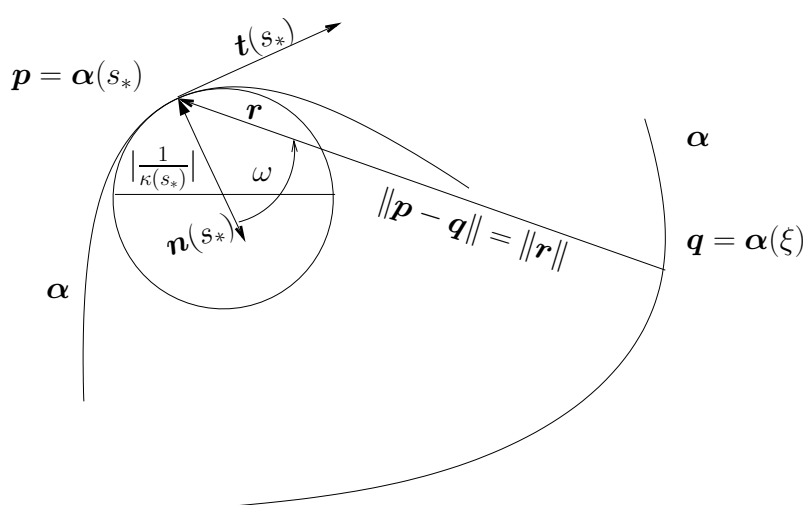

Fig. 2: Local bindings of the view functions. Two portions of the same boundary, the osculating circle, the Frenet frame and the view function $v_{\xi}\left(s_{*}\right)=\|\boldsymbol{r}\|$.

and

$$
\left.\frac{d^{2}}{d s^{2}} v_{\xi}\left(s_{*}\right) \equiv \frac{d^{2}}{d s^{2}} v_{\xi}(s)\right|_{s=s_{*}}=\left.\kappa\left(s_{*}\right) \cos (\omega)\right|_{s=s_{*}}+\left.\frac{\cos ^{2}(\omega)}{\|\boldsymbol{r}\|}\right|_{s=s_{*}}
$$

Even though this proof can be found in [1], it is also provided in Appendix I along with further clarification in the notation. This last proposition relates the planar curvature of $\boldsymbol{\alpha}$ at $\boldsymbol{p}$ to the second derivative of some view function at $s_{*}=\alpha^{-1}(\boldsymbol{p})$ and we notice that, given $\boldsymbol{p}$, the result holds for any view function, since, given $s_{*}$, the choice of $\xi$ is arbitrary. This last observation permits the definition of VAR, as a generalization over the view functions as follows.

Consider $S_{\alpha}$ the set of all the view functions of $\boldsymbol{\alpha}$ and $\|\cdot\|_{v}$ measuring the area below them as:

$$
\|\cdot\|_{v}: S_{\alpha} \rightarrow \mathbb{R}, v_{s} \mapsto\left\|v_{s}\right\|_{v}:=\int_{0}^{\lambda} v_{s}(\xi) d \xi
$$

Definition 2 Let $\varphi_{\alpha}$ be a function defined on $(0, \lambda]$ and taking values in $\mathbb{R}$ as follows:

$$
\varphi_{\alpha}:(0, \lambda] \rightarrow \mathbb{R}: s \mapsto \varphi_{\alpha}(s):=\left\|v_{s}\right\|_{v}
$$

We call $\varphi_{\alpha}$ the View Area Representation or VAR of the curve $\boldsymbol{\alpha} \square$.

VAR is parametrized by the arc length and maps a point $\boldsymbol{\alpha}(s)$ (by means of the arc length parameter $s$ ), to the area $\varphi_{\alpha}(s)$ below the view function $v_{s}$. VAR can be seen as measuring the distance each point has from the rest of the curve, so it increases as we move to remote points on the curve. In the form of a Theorem, we gather results from [1]. Dots represent derivatives always with respect to $s$. 
Theorem 1 Let $\boldsymbol{\alpha} \in C^{3}\left((0, \lambda], \mathbb{R}^{2}\right)$ a closed planar curve of nonzero length $\lambda$, as above. If $\varphi_{\alpha}(s)$ the total distance function (VAR descriptor), $\kappa(s)$ the curvature function and $s_{*}, \xi, \boldsymbol{r}$ and $\omega$ as above, then:

1.

$$
\left.\dot{\varphi}_{\alpha}\left(s_{*}\right) \equiv \frac{d \varphi_{\alpha}(s)}{d s}\right|_{s=s_{*}}=-\left.\int_{0}^{\lambda} \sin (\omega) d \xi\right|_{s=s_{*}}
$$

2.

$$
\left.\ddot{\varphi}_{\alpha}\left(s_{*}\right) \equiv \frac{d^{2} \varphi_{\alpha}(s)}{d s^{2}}\right|_{s=s_{*}}=\kappa\left(s_{*}\right) A\left(s_{*}\right)+B\left(s_{*}\right)
$$

where $A\left(s_{*}\right)=\left.\int_{0}^{\lambda} \cos (\omega) d \xi\right|_{s=s_{*}}$ and $B\left(s_{*}\right)=\left.\int_{0}^{\lambda} \frac{\cos ^{2}(\omega)}{\|\boldsymbol{r}\|} d \xi\right|_{s=s_{*}}$ global shape descriptors measured at $\boldsymbol{\alpha}\left(s_{*}\right)$ and the $\operatorname{dot}(s)$ represent(s) derivative(s) with respect to $s$.

3. If in addition, $\varphi_{\alpha}\left(s_{*}\right)$ a local extremum of $\varphi_{\alpha}(s)$. Then $\kappa\left(s_{*}\right) \neq 0$ and $A\left(s_{*}\right) \neq 0$ and

$$
\kappa\left(s_{*}\right)=\frac{\ddot{\varphi}_{\alpha}\left(s_{*}\right)-B\left(s_{*}\right)}{A\left(s_{*}\right)}
$$

Even though the individual proofs can be found in [1], we also provide them in Appendix II, along with further clarification in the notation. The three equations above are called the GL equations. As we can see from theorem 1 above, the integral descriptor $A$ has a similar interpretation with $\dot{\varphi}$. They both quantify a notion of global displacement of the whole curve with respect to the normal at $s_{*}$. Indeed, if we consider a point $\boldsymbol{\alpha}(\xi)$ that traverses the curve, angle $\omega$ measures the angular displacement of this point with respect to the normal at $s_{*}$. Thus the integral $A$ (and $\dot{\varphi}$ ) can be thought of measuring the total angular displacement of the whole curve with respect to the normal at $s_{*}$. The integral descriptor $B$ has a similar interpretation.

\section{An Algebra of Local Function Behaviors}

We proceed in this section with an algebraic formalism on local function behaviors. The purpose is to simplify the analysis of the GL equations, at least in terms of local extrema localization and magnitude. The focus will be on the localization of zero crossings because of their significance in identifying vertices on curves but could be thought in relation to any local function behavior.

We start by extending the notion of a value of a function at a specific point to that of the set value of the function around this point. Thus, for some function $f$, instead of $f\left(s_{*}\right)$ we now have $f\left(\eta_{s_{*}}\right)$, where $\eta_{s_{*}}$ is an infinitesimal neighborhood around $s_{*}$ and $f\left(\eta_{s_{*}}\right)$ an infinitesimal neighborhood around $f\left(s_{*}\right)$ (continuity and differentiability conditions assumed as usual around $s_{*}$ ). Now we distinguish the following local behaviors for the values of the function at $\eta_{s_{*}}$ (i.d. around $s_{*}$ ): 


\begin{tabular}{||c|c|c|c|c||}
$\oplus$ & $z d c_{f}$ & $z u c_{f}$ & $c z_{f}$ & $n z_{f}$ \\
\hline \hline$z d c_{g}$ & $z d c_{f+g}$ & $z c_{f+g}\left(c z_{f+g}\right)$ & $z d c_{f+g}$ & $n z_{f+g}$ \\
$z u c_{g}$ & $z c_{f+g}\left(c z_{f+g}\right)$ & $z u c_{f+g}$ & $z u c_{f+g}$ & $n z_{f+g}$ \\
$c z_{g}$ & $z d c_{f+g}$ & $z u c_{f+g}$ & $c z_{f+g}$ & $n z_{f+g}$ \\
$n z_{g}$ & $n z_{f+g}$ & $n z_{f+g}$ & $n z_{f+g}$ & $n z_{f+g}\left(z c_{f+g}, c z_{f+g}\right)$ \\
\hline
\end{tabular}

Table 1: Local algebra for the $\oplus$ operation.

(i) Zero crossing at $\eta_{s_{*}}$, we write it as $z c_{f\left(s_{*}\right)}$ when the function's $f$ order of contact with the horizontal axis, around $s_{*}$, is exactly one. This has subcases that will be signified by $z d c$ and $z u c$, meaning zero down crossing and zero up crossing respectively, with the obvious interpretations whenever their distinction plays a role. We will occasionally use $z c$ to refer to both of them simultaneously.

(ii) Constant zero at $\eta_{s_{*}}$, we write it as $c z_{f\left(s_{*}\right)}$. when the function's order of contact with the horizontal axis in an infinitesimal neighborhood around $s_{*}$ is greater or equal to 2 ; All the following cases are $c z$ at $\eta_{s_{*}}$ :

- The function attains a zero local extremum at $s_{*}$.

- The function attains a saddle stationary point at $s_{*}$ that equals zero.

- The function is constant around $s_{*}$ and equals zero.

(iii) Non zero at $\eta_{s_{*}}$, we write it as $n z_{f\left(s_{*}\right)}$ when the function's order of contact with the horizontal axis in an infinitesimal neighborhood around $s_{*}$ is zero.

The above local behaviors form the set $L B \equiv\left\{l b_{i}\right\}=\{z u c, z d c, c z, n z\}$, called the set of local function behaviors and are mutually exclusive and collectively exhaustive in that sense.

Since the local behaviors are considered for a function at a specific point we can consider them as an $l$ functoid defined on $C^{3}((0, \lambda], \mathbb{R}) \times(0, \lambda]$ and taking values in $L B$ thus:

$$
\begin{aligned}
l & : C^{3}((0, \lambda], \mathbb{R}) \times(0, \lambda] \rightarrow L B \\
& :(f, s) \mapsto l(f, s) \equiv l\left(f\left(\eta_{s}\right)\right)
\end{aligned}
$$

the local behavior of function $f$ around $s$. Through $l$ we define a local point-wise addition of local behaviors as:

$$
\begin{aligned}
& \oplus: L B \times L B \rightarrow L B: \\
& (l(f, s), l(g, s)) \mapsto l(f, s) \oplus l(g, s) \equiv(l(f+g), s)
\end{aligned}
$$

thus the $\oplus$ of the local behaviors of $f$ and $g$ at $s$ is the local behavior of $f+g$ at $s$.

Similarly, a local point-wise multiplication is defined:

$$
\begin{aligned}
& \otimes: L B \times L B \rightarrow L B: \\
& (l(f, s), l(g, s)) \mapsto l(f, s) \otimes l(g, s) \equiv(l(f g), s)
\end{aligned}
$$

One can verify that the rules of Table 1 hold for $\oplus$. For the multiple cases, the extra entries in parenthesis are exceptions. These depend on the slope and the specific values of the functions at and around $s_{*}$ but they are special cases 
and will be treated accordingly. Similarly, the rules of Table 2 hold for $\otimes$. There, $f, g, f g$ are easily assumed and thus omitted for economy of space.

One could be tempted to see the above as an algebra of 1st order Taylor approximants, since any $C^{1}$ function can be approximated near the argument $x=x_{0}$ by a linear function of the kind $k\left(x-x_{0}\right)+d$ and the cases $n z, z d c, z u c, c z$ correspond to $d \neq 0, d=0$ and $k<0, d=0$ and $k>0$, and $k=d=0$, respectively. Addition and multiplication and subsequent 1st order Taylor expansion are the analogous to the above algebraic operations. Such an approximation however wouldn't have been adequate for further analysis, since of interest here is not an approximation but rather the exact localization of zero crossings in linear combinations of functions of well known local behaviors. We thus proceed likewise.

So far $\oplus$ has been considered through local point-wise operations of functions around a common saturation point. We now extend this local operation to allow cases of different saturation points for the two functions. Thus:

\section{Definition 3}

$$
\begin{aligned}
& \oplus: L B \times L B \rightarrow L B \\
& \quad:\left(l\left(f, s_{1}\right), l\left(g, s_{2}\right)\right) \mapsto\left(l(f+g), s_{*}\right)
\end{aligned}
$$

where $s_{*}$ is the closest to $s_{1}$ point in $\left[s_{1}, s_{2}\right]$ for which it holds that $\mid f\left(s_{*}\right)+$ $g\left(s_{*}\right) \mid$ is a global minimum of $|f(s)+g(s)|, s \in\left[s_{1}, s_{2}\right]$ and $l\left(f+g, s_{*}\right)$ is the local behavior of $(f+g)(s)$ around $(f+g)\left(s_{*}\right) \square$

We can verify that if $s_{1}=s_{2}$ then $s_{*}=s_{1}=s_{2}$ and the case reduces to the point-wise behavior around a common saturation point already examined above. But the above definition extends the capability of the $\oplus$ operation to consider different saturation points for the local behavior of the two functions taking part in $\oplus$, in a way that favors localization of new zero crossings. Indeed, it is easy to see that according to the above definition: $z c_{f}\left(s_{1}\right) \oplus z c_{g}\left(s_{2}\right)=$ $z c_{f+g}\left(s_{*}\right)$ iff $\left[s_{1}, s_{2}\right]$ is an interval where $f$ and $-g$ intercept each other at $s_{*} \in\left[s_{1}, s_{2}\right]$.

For the $\otimes$ operation an analogous extension is not necessary but what is important to notice about $\otimes$ is that $z c_{f}\left(s_{*}\right) \otimes n z_{g}\left(s_{*}\right)=z c_{f g}\left(s_{*}\right)$ and that if $s_{1}=s_{*}-\epsilon, s_{2}=s_{*}+\epsilon, \lim _{s \rightarrow s_{1}} f(s)= \pm \infty, \lim _{s \rightarrow s_{2}} f(s)=\mp \infty$ and $g$ bounded in $\left(s_{1}, s_{2}\right)$ then $\lim _{s \rightarrow s_{1}} f g(s)= \pm \infty$ and $\lim _{s \rightarrow s_{2}} f g(s)=\mp \infty$ (or $\lim _{s \rightarrow s_{1}} f g(s)=\mp \infty$ and $\lim _{s \rightarrow s_{2}} f g(s)= \pm \infty$ depending on the sign of $g$ around $s_{*}$ ).

In words this last one states that if the absolute values of the zero crossing participant in the $\otimes$ operation increase without bound around $s_{*}$ but the non zero participant is bounded around $s_{*}$, then the resulting from the $\otimes$ operation behavior is a zero crossing with its absolute values increasing without bound around $s_{*}$. This observation is important because it affects the localization of the resulting from $\oplus$ zero crossing as the following Proposition states: 
Proposition 2 If $l\left(f, s_{*}\right)$ is a zero crossing $(z c)$ of $f$ at $s_{*}$ and $l(g, s)$ an arbitrary local behavior (lb) of $g$ at $s$, also $s_{1}=s_{*}-\epsilon, s_{2}=s_{*}+\epsilon$ such that $\lim _{s \rightarrow s_{1}} f(s)= \pm \infty$ and $\lim _{s \rightarrow s_{2}} f(s)=\mp \infty, f$ continuous in $\left(s_{1}, s_{2}\right), g$ continuous and bounded in $\left(s_{1}, s_{2}\right)$ then: $\lim _{s_{1} \rightarrow s_{*}}\left(z c_{f}\left(s_{*}\right) \oplus l b_{g}\left(s_{1}\right)\right)=\lim _{s_{2} \rightarrow s_{*}}\left(z c_{f}\left(s_{*}\right) \oplus\right.$ $\left.l b_{g}\left(s_{2}\right)\right)=z c_{f+g}\left(s_{*}\right)$.

Proof We first pick the closest to $s_{1}$ point $s_{0} \in\left(s_{1}, s_{2}\right)$ such that $f\left(s_{0}\right)=$ $-g\left(s_{0}\right)$. Such a point always exists since the values of $f$ increase/decrease inversely without bound near $s_{1}$ and $s_{2}$ and $g$ bounded in $\left(s_{1}, s_{2}\right)$. At this point $s_{0}, f+g$ has a zero crossing but as $s_{1} \rightarrow s_{*}$ and $s_{2} \rightarrow s_{*}$ then also $s_{0} \rightarrow s_{*}$ from either side and the proof is complete.

In words this proposition states that as the absolute values of $f$ increase around $s_{*}$ then the location at which $f \oplus g$ achieves a zero crossing approaches $s_{*}$ and it is a key proposition since it permits elimination of the bounded member in $\oplus$ (in terms of zero crossing localization), when the conditions of high values around the zero crossing (e.g. by means of the observation regarding $\otimes$ above) hold for the unbounded member.

\section{Zero Crossing Analysis of the second Global Local Equation}

Lets visit again equation (9) which links $\kappa$ to $\ddot{\varphi}$ up to global descriptors $A$ and $B$. All the quantities on the right hand side are integrals defined on the whole of the shape. They don't change significantly with noise. This definition of curvature is thus stronger than the traditional one in the sense that is not directly exposed to noise.

While this equation permits calculating $\kappa$ even in the presence of noise, a concern here will be whether it can be further simplified, if we are only looking for vertices. If further simplification of equation (9) can lead to a faster/simpler method of estimating vertices is significant because vertices summarize the perceptual importance of curvature, while at the same time traditional local methods cannot compute vertices in the presence of noise. This exploration will lead us to a surprising result, as we will discover that the rate of change of $A$ contributes a location aspect to the calculation of vertices. Assuming usual valid continuity and differentiability conditions, we start with the derivative of $A$ at the origin of the Frenet frame $s_{*}$ :

$$
\begin{aligned}
\left.\dot{A}\left(s_{*}\right) \equiv \frac{d A(s)}{d s}\right|_{s=s_{*}} & =\left.\frac{d}{d s}\left(\int_{0}^{\lambda} \cos (\omega) d \xi\right)\right|_{s=s_{*}} \\
& =\left.\int_{0}^{\lambda} \frac{\partial}{\partial s} \cos (\omega) d \xi\right|_{s=s_{*}} \\
& =-\left.\int_{0}^{\lambda} \dot{\omega} \sin (\omega) d \xi\right|_{s=s_{*}}
\end{aligned}
$$


From the combination of (3) and (4) we get:

$$
-\frac{\partial}{\partial s} \sin (\omega)=-\dot{\omega} \cos (\omega)=\cos (\omega)\left(\kappa(s)+\frac{\cos (\omega)}{\|\boldsymbol{r}\|}\right)
$$

and thus for $\cos (\omega) \neq 0$ we get:

$$
-\dot{\omega}=\kappa(s)+\frac{\cos (\omega)}{\|\boldsymbol{r}\|}
$$

Substituting $\dot{\omega}$ from above to (14):

$$
\begin{aligned}
\left.\dot{A}\left(s_{*}\right) \equiv \dot{A}(s)\right|_{s=s_{*}} & =\kappa\left(s_{*}\right)\left(\left.\int_{0}^{\lambda} \sin (\omega) d \xi\right|_{s=s_{*}}\right)+ \\
& \left.\int_{0}^{\lambda} \frac{\sin (\omega) \cos (\omega)}{\|\boldsymbol{r}\|} d \xi\right|_{s=s_{*}}
\end{aligned}
$$

and from equation (7):

$$
\dot{A}\left(s_{*}\right)=-\kappa\left(s_{*}\right) \dot{\varphi}\left(s_{*}\right)+\left.\int_{0}^{\lambda} \frac{\sin (\omega) \cos (\omega)}{\|\boldsymbol{r}\|} d \xi\right|_{s=s_{*}}
$$

This last equation reveals an unexpected behavior of $\dot{A}$. It states that local extrema locations of $A$ and $\varphi$ approach each other as $|\kappa|$ increases. To see this we need to recall Proposition 2 and notice that the integral at the far right side of equation (18) is constant with respect to $\kappa$. According then to Proposition 2, the location at which $\dot{A}$ achieves a zero crossing can be arbitrarily close to a zero crossing of $\dot{\varphi}\left(s_{*}\right)$ as $\left|\kappa\left(s_{*}\right)\right|$ increases. This result is encouraging because it links $\dot{A}$ with $\dot{\varphi}$ in terms of zero crossing analysis and thus may lead to a further simplification of equation (9) as was initially pursued. We continue in hopes that similar conclusions hold for $\dot{B}$ :

$$
\begin{aligned}
& \left.\dot{B}\left(s_{*}\right) \equiv \frac{d B(s)}{d s}\right|_{s=s_{*}}=\left.\frac{d}{d s}\left(\int_{0}^{\lambda} \frac{\cos ^{2}(\omega)}{\|r\|} d \xi\right)\right|_{s=s_{*}} \\
& =\left.\int_{0}^{\lambda} \frac{\partial}{\partial s} \frac{\cos ^{2}(\omega)}{\|r\|} d \xi\right|_{s=s_{*}}=-\left.2 \int_{0}^{\lambda} \frac{\dot{\omega} \cos (\omega) \sin (\omega)}{\|r\|} d \xi\right|_{s=s_{*}}+ \\
& +\left.\left.\int_{0}^{\lambda} \frac{\cos ^{2}(\omega) \sin (\omega)}{\|r\|^{2}} d \xi\right|_{s=s_{*}} \stackrel{(15)}{=} 2 \kappa\left(s_{*}\right) \int_{0}^{\lambda} \frac{\cos (\omega) \sin (\omega)}{\|r\|} d \xi\right|_{s=s_{*}}+ \\
& +\left.2 \int_{0}^{\lambda} \frac{\cos ^{2}(\omega) \sin (\omega)}{\|r\|^{2}} d \xi\right|_{s=s_{*}}+\left.\int_{0}^{\lambda} \frac{\cos ^{2}(\omega) \sin (\omega)}{\|r\|^{2}} d \xi\right|_{s=s_{*}} \\
& =\left.2 \kappa\left(s_{*}\right) \int_{0}^{\lambda} \frac{\cos (\omega) \sin (\omega)}{\|r\|} d \xi\right|_{s=s_{*}}+ \\
& +\left.3 \int_{0}^{\lambda} \frac{\cos ^{2}(\omega) \sin (\omega)}{\|r\|^{2}} d \xi\right|_{s=s_{*}}
\end{aligned}
$$


$\dot{B}$ doesn't seem to follow a similar pattern but we proceed further anyways. Starting from (8) and assuming valid continuity and differentiability conditions, we take derivatives on both sides with respect to $s$ :

$$
\dddot{\varphi}_{\alpha}\left(s_{*}\right)=A\left(s_{*}\right) \dot{\kappa}\left(s_{*}\right)+\kappa\left(s_{*}\right) \dot{A}\left(s_{*}\right)+\dot{B}\left(s_{*}\right)
$$

and by means of equations (18) and (19):

$$
\begin{aligned}
& \dddot{\varphi}\left(s_{*}\right)=\dot{\kappa}\left(s_{*}\right) A\left(s_{*}\right)-\kappa^{2}\left(s_{*}\right) \dot{\varphi}\left(s_{*}\right)+ \\
& +3 \kappa\left(s_{*}\right)\left(\left.\int_{0}^{\lambda} \frac{\cos (\omega) \sin (\omega)}{\|r\|} d \xi\right|_{s=s_{*}}\right)+ \\
& +\left.3 \int_{0}^{\lambda} \frac{\cos ^{2}(\omega) \sin (\omega)}{\|r\|^{2}} d \xi\right|_{s=s_{*}}
\end{aligned}
$$

We realize that if we rewrite 21 as:

$$
\begin{aligned}
& \dddot{\varphi}\left(s_{*}\right)=\dot{\kappa}\left(s_{*}\right) A\left(s_{*}\right) \\
& -\kappa\left(s_{*}\right)\left(\kappa\left(s_{*}\right) \dot{\varphi}\left(s_{*}\right)-\left.3 \int_{0}^{\lambda} \frac{\cos (\omega) \sin (\omega)}{\|r\|} d \xi\right|_{s=s_{*}}\right) \\
& +\left.3 \int_{0}^{\lambda} \frac{\cos ^{2}(\omega) \sin (\omega)}{\|r\|^{2}} d \xi\right|_{s=s_{*}}
\end{aligned}
$$

we can proceed with a zero crossing analysis according to the theory established in section 5 . Indeed, at a zero crossing $\dot{\varphi}\left(\eta_{s_{*}}\right)$ and as $\left|\kappa\left(s_{*}\right)\right| \rightarrow \infty$, equation (22) can be now examined at a zero crossing basis as:

$$
\begin{aligned}
& \dddot{\varphi}\left(\eta_{s_{*}}\right) \equiv \dot{K}\left(\eta_{s_{*}}\right) \otimes A\left(\eta_{s_{*}}\right) \oplus-K\left(\eta_{s_{*}}\right) \\
& \otimes\left[\left(K\left(\eta_{s_{*}}\right) \otimes \dot{\varphi}\left(\eta_{s_{*}}\right)\right) \oplus-\left.3 \int_{0}^{\lambda} \frac{\cos (\omega) \sin (\omega)}{\|r\|} d \xi\right|_{s \in \eta_{s_{*}}}\right] \\
& \left.\oplus 3 \int_{0}^{\lambda} \frac{\cos ^{2}(\omega) \sin (\omega)}{\|r\|^{2}} d \xi\right|_{s \in \eta_{s_{*}}}
\end{aligned}
$$

with $K\left(\eta_{s_{*}}\right)$ meaning $\kappa$ approaching infinity at a neighborhood of $s_{*}$. At this point the reader may notice the conceptual transition in (23) becoming an identity between local behaviors. Symbol $\equiv$ reminds it is not an equality between values any more. We may also recognize in the operation in the square brackets a case of Proposition (2). Indeed, as $\left|\kappa\left(s_{*}\right)\right| \rightarrow \infty$ the absolute values around zero of the zero crossing signified by $K\left(\eta_{s_{*}}\right) \otimes \dot{\varphi}\left(\eta_{s_{*}}\right)$ increase without bound while the other term $\left.3 \int_{0}^{\lambda} \frac{\cos (\omega) \sin (\omega)}{\|r\|} d \xi\right|_{s \in \eta_{s_{*}}}$ is bounded in $\eta_{s_{*}}$. In terms of zero crossing localization, the integral term can be omitted since, as 


\begin{tabular}{||c|c|c|c|c||}
$\otimes$ & $z d c$ & $z u c$ & $c z$ & $n z$ \\
\hline \hline$z d c$ & $c z$ & $c z$ & $c z$ & $z c$ \\
$z u c$ & $c z$ & $c z$ & $c z$ & $z c$ \\
$c z$ & $c z$ & $c z$ & $c z$ & $c z$ \\
$n z$ & $z c$ & $z c$ & $c z$ & $n z$ \\
\hline
\end{tabular}

Table 2: Local algebra for the $\otimes$ operation.

already explained, the zero crossing location is dominated by the local term, thus:

$$
\begin{aligned}
& \dddot{\varphi}\left(\eta_{s_{*}}\right) \equiv\left[\dot{K}\left(\eta_{s_{*}}\right) \otimes A\left(\eta_{s_{*}}\right)\right] \oplus \\
& {\left[-\left.K^{2}\left(\eta_{s_{*}}\right) \otimes \dot{\varphi}\left(\eta_{s_{*}}\right) \oplus 3 \int_{0}^{\lambda} \frac{\cos ^{2}(\omega) \sin (\omega)}{\|r\|^{2}} d \xi\right|_{s \in \eta_{s_{*}}}\right]}
\end{aligned}
$$

and for the same reasons finally:

$$
\dddot{\varphi}\left(\eta_{s_{*}}\right) \equiv\left[\dot{K}\left(\eta_{s_{*}}\right) \otimes A\left(\eta_{s_{*}}\right)\right] \oplus\left[-K^{2}\left(\eta_{s_{*}}\right) \otimes \dot{\varphi}\left(\eta_{s_{*}}\right)\right]
$$

This last equation describes a localization mechanism based on the interaction between zero crossings of functions that are easy to calculate.

\section{Global Vertices and the Concept of Noising}

In equation (25) a mechanism of localizing zero crossings of $\dddot{\varphi}\left(\eta_{s_{*}}\right)$ based on the local behavior of meaningful local $(k, \dot{k})$ and global $(A, \dot{\varphi})$ descriptors is established. Localizing zero crossings of $\dddot{\varphi}\left(\eta_{s_{*}}\right)$, if compared to solving analytical in terms of $\dddot{\varphi}(s)$, not only is easier (given the tools in section (5)) but in our case, is also of a greater practical value, because vertex analysis is now turned into a search for local extrema locations in scalar functions which is an easier task unaffected by noise. But there is another unexpected benefit in the formulation of equation (25). The requirement for increasing $\kappa$ can be met by the presence of noise and thus noise is turned into a facilitating factor for localizing vertices according to equation (25). First we show that the location of the curve's vertices of significant curvature can be inferred from the extrema of $\varphi$. This is formally proven in Proposition (3).

Proposition 3 Let $\boldsymbol{\alpha} \in C^{3}\left((0, \lambda], \mathbb{R}^{2}\right)$ a closed planar curve of length $\lambda \neq 0$ as usual, $\varphi_{\alpha}(s)$ the total distance function (VAR descriptor), and $\kappa(s)$ the curvature function. If $\varphi_{\alpha}(s)$ achieves a local extremum in $\eta_{s_{*}}$ in such a way that $\dot{\varphi}_{\alpha}\left(\eta_{s_{*}}\right)$ is a zero crossing of $\dot{\varphi}_{\alpha}(s)$ and $\dddot{\varphi}_{\alpha}\left(\eta_{s_{*}}\right)$ a zero crossing of $\dddot{\varphi}_{\alpha}(s)$ then as $k\left(s_{*}\right) \rightarrow \infty$ there exists a vertex of $\boldsymbol{\alpha}$ in $\boldsymbol{\alpha}\left(\eta_{s_{*}}\right)$.

Proof Since $\varphi_{\alpha}\left(s_{*}\right)$ is an extreme of $\varphi_{\alpha}(s)$ in a way that both $\dddot{\varphi}_{\alpha}\left(\eta_{s_{*}}\right)$ and $\dot{\varphi}_{\alpha}\left(\eta_{s_{*}}\right)$ are zero crossings of $\dddot{\varphi}_{\alpha}(s)$ and $\dot{\varphi}_{\alpha}(s)$ respectively, from Theorem (1) we get that $\kappa\left(s_{*}\right) \neq 0$ therefore $K^{2}\left(\eta_{s_{*}}\right) \otimes \dot{\varphi}_{\alpha}\left(\eta_{s_{*}}\right)$ is a zero crossing. This according to equation (25) means that $\dot{K}\left(\eta_{s_{*}}\right) \otimes A\left(\eta_{s_{*}}\right)$ will have to allow one of the following cases: 
- $\dot{K}\left(\eta_{s_{*}}\right) \otimes A\left(\eta_{s_{*}}\right)$ is also a zero crossing. But since from Theorem (1), $A\left(s_{*}\right) \neq$ 0 we conclude that $\dot{K}\left(\eta_{s_{*}}\right)$ is a zero crossing and in $\boldsymbol{\alpha}\left(\eta_{s_{*}}\right)$ there is a vertex of $\boldsymbol{\alpha}$.

- $\dot{K}\left(\eta_{s_{*}}\right) \otimes A\left(\eta_{s_{*}}\right)$ is locally zero. But again from Theorem (1), $A\left(s_{*}\right) \neq 0$ thus $\dot{K}\left(\eta_{s_{*}}\right)$ is locally zero. Since also from Theorem $(1) \kappa\left(s_{*}\right) \neq 0$, we conclude that $\boldsymbol{\alpha}(s)$ has locally constant non zero curvature at $\eta_{s_{*}}$ thus in $\boldsymbol{\alpha}\left(\eta_{s_{*}}\right)$ there is a vertex of $\boldsymbol{\alpha}$.

So in both of the above cases in $\boldsymbol{\alpha}\left(\eta_{s_{*}}\right)$ there is a vertex of $\boldsymbol{\alpha}$ and the proof is complete. $\square$

Vertices according to Proposition 3 are localized natively, not relying on smooth curvature calculations. In the collocation of $\dot{\varphi}$ and $\dddot{\varphi}$ zero crossings and subject to the assumptions of equation (25), Proposition 3 establishes sufficient conditions for the existence of certain vertices. This development provides guidance for the synthesis of a vertex identification method in the context of the previous analytical approach. This is outlined in section (7.2) but first the important for our approach concept of Global Vertices is introduced in section (7.1).

\subsection{Global Vertices}

One wonders how much noise is enough to make equation (25) valid and whether the fact that $|\kappa|$ increases everywhere (after existing or induced noise on the curve) obscures the underlying shape. For the latter question, in Fig.(4) a discrete case is demonstrated, where additive perturbations induced in a principled manner increases the curvature of the resulting curve at all of its points, but at the same time retains the initial curvature information of the initial curve. The continuous case can be similarly inferred if instead of a piecewise curve one considers infinitesimal arc elements in a suitable manner. For the former question the answer lies with equations (23) and (24) where the terms $C=\left.3 \int_{0}^{\lambda} \frac{\cos (\omega) \sin (\omega)}{\|r\|} d \xi\right|_{s \in \eta_{s_{*}}}$ and $D=\left.3 \int_{0}^{\lambda} \frac{\cos ^{2}(\omega) \sin (\omega)}{\|r\|^{2}} d \xi\right|_{s \in \eta_{s_{*}}}$ have to be compensated by the slope of $\dot{\varphi}\left(\eta_{s_{*}}\right)$ and the magnitude of $K\left(s_{*}\right)$ and $K^{2}\left(s_{*}\right)$, respectively. The continuity of all functions involved in the above analysis guaranties a smooth transition of zero crossings as $\kappa$ increases against $C$ and $D$ and permits a valid extension from infinitesimal to finite neighborhoods around $s_{*}$ while keeping the validity of all the previous results. We can thus permit $\eta_{s_{*}}$ to aquire finite length and become $H_{s_{*}}$, signifying a compact interval of finite nonzero length around $s_{*}$ such that $\dot{\varphi}\left(H_{s_{*}}\right)$ and $A\left(H_{s_{*}}\right)$ exhibits the same local behavior with $\dot{\varphi}\left(\eta_{s_{*}}\right)$ and $A\left(\eta_{s_{*}}\right)$ respectively around $s_{*}$. We can now see again equation (25) as:

$$
\dddot{\varphi}\left(H_{s_{*}}\right) \equiv\left[\dot{\kappa}_{H_{s_{*}}} \otimes A\left(H_{s_{*}}\right)\right] \oplus\left[-\kappa_{H_{s_{*}}}^{2} \otimes \dot{\varphi}\left(H_{s_{*}}\right)\right]
$$

which is always valid as long as $\kappa$ is a function of the length of $H_{s_{*}}$ which in turn is a function of $C$ and $D$. Indeed, one can think in an inverse manner and 
Vertex Localization-SmoothShape

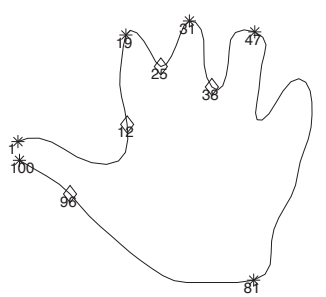

Zero crossings of $\mathrm{d} \phi / \mathrm{ds}$ and $\mathrm{d}^{3} \phi / \mathrm{ds} \mathrm{s}^{3}$
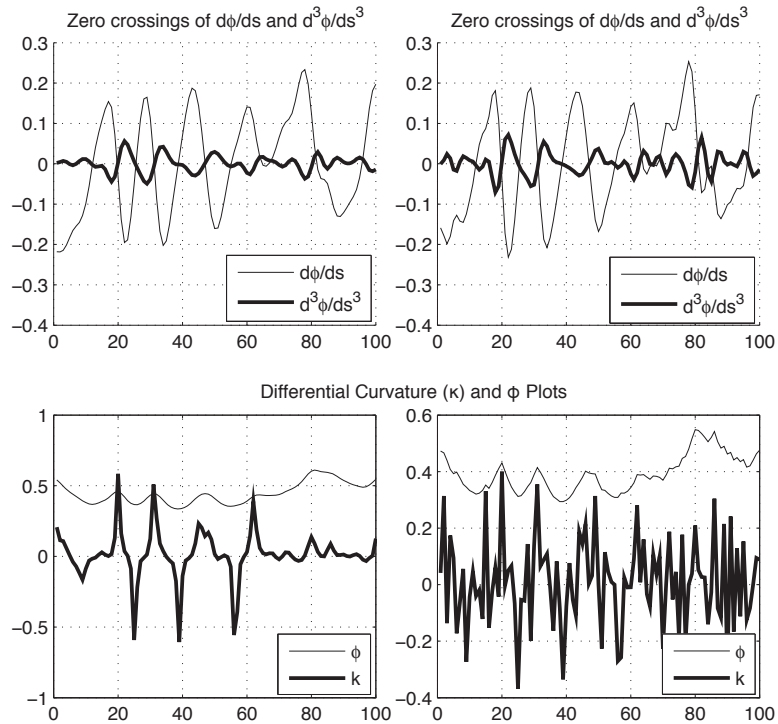

Fig. 3: Global Vertex localization for smooth and noise versions of the same Kimia Silhouette using co- localization of $\phi$ and $\ddot{\phi}$ extreme points. Stars and diamonds are curvature's local maxima and minima respectively. Points are marked on the shapes for every 10th point in a total of 100 points per shape and are also assumed as the $\mathrm{x}$ axis in all plots. The co-localization of zero crossings that appear in the second row of plots are validated against $\phi$ and curvature plots appearing in the last row. We notice that more points are correctly identified in the noisy version. We also notice that the proposed method produces correct results even though the differential curvature descriptor has collapsed in the noisy case.

ask that given $s_{*}$ (as a zero crossing location of $\dot{\varphi}(s)$ ), $C, D$ and $H_{s_{*}}$ as a finite neighborhood around $s_{*}$, what is the minimum $\kappa$ that would guarantee a zero crossing of $\dddot{\varphi}(s)$ in $H_{s_{*}}$ ? The answer to this question is a finite curvature, thus the requirement of $k\left(s_{*}\right) \rightarrow \infty$ in order of the zero crossings of $\dot{\varphi}(s)$ and $\dddot{\varphi}(s)$ to coexist in an infinitesimal neighborhood $\eta_{s_{*}}$, can been relaxed to that of a 


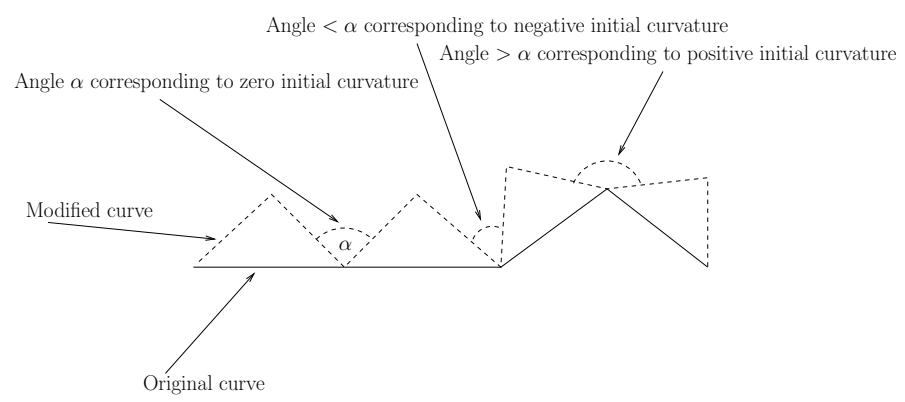

Fig. 4: The concept of additive perturbations is illustrated here. New points are added to the contour but the initial curvature information is retained.

finite curvature $\kappa_{H_{s_{*}}}$ that would guarantee the existence of both zero crossings of $\dot{\varphi}(s)$ and $\dddot{\varphi}(s)$ in $H_{s_{*}}$ and thus, according to Proposition (3), a vertex of $\boldsymbol{\alpha}$, in the finite neighborhood $H_{s_{*}}$. The finite neighborhood $H_{s_{*}}$ is conceptualized in relation to the infinitesimal neighborhood $\eta_{s_{*}}$ and the local behavior of the pertinent functions in $\eta_{s_{*}}$. Thus, in $C$ and $D$ we have a measure of the amount of perturbation $\left(\kappa_{H_{*}}\right)$ needed at $s_{*}$ to bring in equation (26) or equivalently the tolerance in the collocation of the zero crossings of $\dot{\varphi}(s)$ and $\dddot{\varphi}(s)$ one can assume to infer the existence of a vertex for given values of $\kappa_{H_{*}}$. We are now in position to define Global Vertices.

Definition 4 A Global Vertex $\boldsymbol{\alpha}\left(s_{*}\right)$ is a vertex of $\boldsymbol{\alpha}(s)$ for which it holds that $\dot{\varphi}_{\alpha}\left(\eta_{s_{*}}\right)$ is a zero crossing of $\dot{\varphi}_{\alpha}(s) \square$.

According to this definition a Global Vertex is a vertex but not all vertices are Global Vertices. Similarly, the condition $\dot{\varphi}_{\alpha}\left(s_{*}\right)=0$ is not sufficient to guarantee a vertex (and thus a global vertex) at $s_{*}$. From the above analysis one can infer that at points where $C=D=0$ a vertex (and thus a global vertex) can be guaranteed at $s_{*}$ when both $\dot{\varphi}_{\alpha}\left(\eta_{s_{*}}\right)$ and $\dddot{\varphi}_{\alpha}\left(\eta_{s_{*}}\right)$ are zero crossings. In the general case however, where $C \neq 0, D \neq 0$, a vertex (and thus a global vertex) can be guaranteed in $H_{s_{*}}$ when after $K_{H_{s_{*}}}$ is applied at $s_{*}$ both $\dot{\varphi}_{\alpha}\left(H_{s_{*}}\right)$ and $\dddot{\varphi}_{\alpha}\left(H_{s_{*}}\right)$ are zero crossings, where $H_{s_{*}}$ is proportional to $C$ and $D$ and $K_{H_{s_{*}}}$ a function of $H_{s_{*}}$. The above analysis leads to the concept of noising as an enabler for Global Vertex identification and is formally presented in the next section 7.2 .

\subsection{Noise as an Enabler}

The following noising algorithm is now designed, consisting of controlled perturbations on the boundary of noisy or smooth shapes. This process can be performed in an additive manner to the existing boundary, not affecting the initial boundary points. In the discrete case of a digital curve, for each pair of 


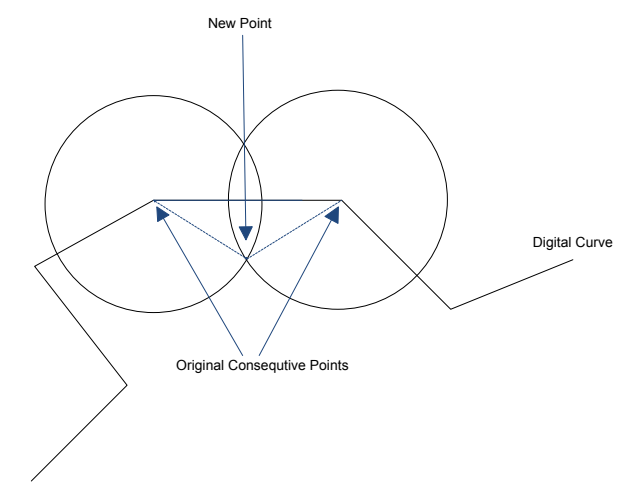

Fig. 5: The method of noising as opposed to smoothing is illustrated here. New points are added to the contour with the purpose to enrich the tangent directions around the original points. This will facilitate the proposed method in identifying vertices.

consecutive points on the initial boundary, a new point will be added at the intersection of the circles centered at the original points and having equal radii of a certain length, greater than half the distance between the two original points (Fig.5). Noise applied in this principled way has three global consequences, all beneficial to the proposed global method (Fig.4):

- The absolute local curvature at each point on the curve increases, thus equation (26) can be appropriately applied.

- The relative rate of change of each point's total distance to the rest of the curve is not changed significantly, thus global curvature calculations are also not affected.

- The tangent directions around each point are enriched. This helps $\dot{\varphi}$ to identify remote points on the curve and $\dddot{\varphi}$ to measure the relative change of their distance to the rest of the curve. Native Global Vertex identification according to Proposition (3) is thus facilitated.

One can then realize that the negative local effects of noise have turned into positive global effects. For the proposed method in particular, noising is an enabler through equation (26) and according to Proposition (3). This emerges as a paradox, since vertices are differentials of a higher order than curvature, thus even more sensitive to noise than curvature is with traditional methods.

It is important to notice that Global Vertices are detected directly without the need for curvature calculations. In other words, the method does not rely on a smooth estimation of curvature around vertices, albeit them being of a higher differential order than curvature. Another issue worth noticing, is that the above procedure of noising can be applied recursively to form neighborhoods of increasing differential order around the initial curve points, resulting in an analogous concept to that of incremental smoothing. Noising, as a preprocessing step for global methods, may be viewed as a conceptual duality to what smoothing is for local methods. 
Noisy Shape

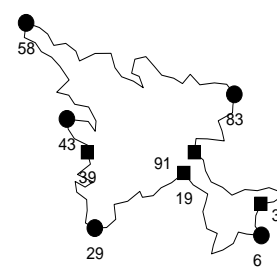

Smooth Version

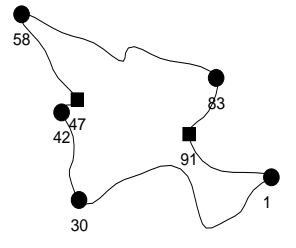

Global Vertex Estimation
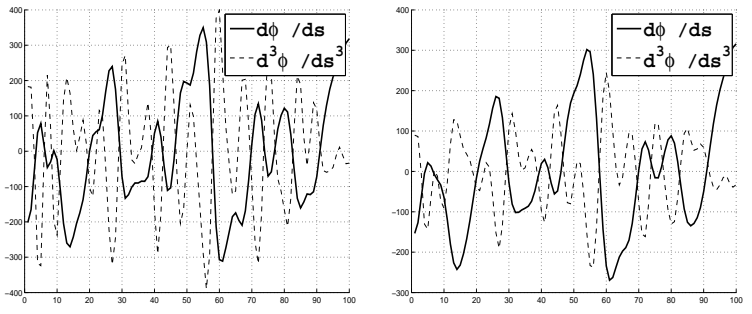

Global Curvature Estimation
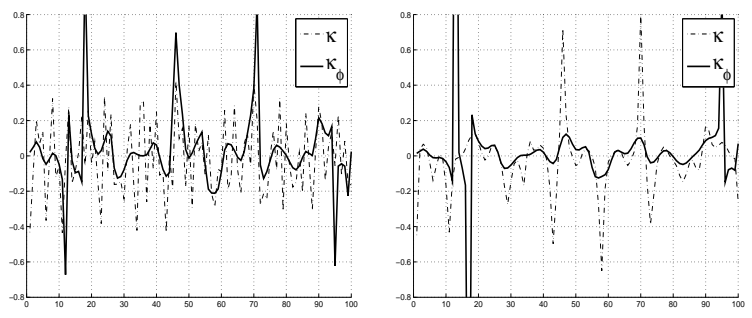

Fig. 6: Curvature estimation and Global Vertex localization of a typical KIMIA silhouette. At the top row of plots, solid circles and squares are curvature local minima and maxima respectively, identified by the proposed method for smooth and noisy versions of the same KIMIA silhouette. Compare these to the stars and diamonds identified for the same shapes by the LAII method in Fig.(9). At the middle row of plots, $\phi$ and $\phi$ are shown, the collocation of which at a zero crossing, defines vertices according to the proposed method. At the bottom row of plots we see the differential curvature $(\kappa)$ and the curvature estimator $\kappa_{\phi}$ (based on equation (9)), valid only at points where $A \neq 0$, for both noisy and smooth shapes. Notice also the robustness of the proposed estimator in the noisy case where the differential curvature has collapsed. 
In the experimental section that follows, the above methods for curvature calculation (according to (9)) and vertex localization (according to Proposition (3)) are validated. A comparison to LAII[26] in these tasks, for smooth and noisy versions of shapes is also presented.

\section{Experimental Validation with additive Gaussian Noise}

In this section the theoretical findings above are validated with experiments. First we demonstrate the localization of Global Vertices and how it is improved by noise. Then, in (8.2), in the absence of other global method for vertex localization, we compare with LAII[26], as the most robust among other local methods. LAII is a localized method primarily for noise resistant curvature calculations. By mean of integrals, LAII defines certain 2D circles around every point on the curve. These circles absorb noise by providing robust curvature estimations in a certain localized sense. If seen as a robust curvature method that uses integrals, LAII is comparable to the proposed herein global method. Vertices however, according to LAII, will be localized as curvature extrema according to the traditional differential definition, whereas in the proposed approach Global Vertices are disconnected from curvature, the main contribution of [25] that is also used in this paper. In the absence of other global method, comparisons are performed to LAII in both curvature calculations and vertex localization in noisy and smooth shapes. The experiments show that while noise is absorbed and its negative effects are minimized in LAII, the proposed global approach actually benefits from noise in vertex localization. As noise increases LAII's (and any other local method's in that respect) effect is in minimizing the negative local effects of noise, while the proposed method gains better performance by exploiting the positive global effects of noise. Curvature calculations according to the global method are also not affected significantly by noise. The comparison to LAII reveals that the advantages of the proposed approach in both vertex identification and curvature estimation stem from the global conceptual approach, a fact that turned noise on our side. Another important advantage, unique to the proposed global method with regard to LAII and other local methods is in differentiating vertices with respect to context. Indeed, local methods, as was discussed in the introductory section, have an inherent deficiency to incorporate context. The proposed method on the other hand, demonstrates how context can be conceptualized naturally when global approaches are employed.

\subsection{Vertex Localization and the Effect of Noise}

In Fig.(3) a noisy silhouette is compared to its smoothed version. There, we see that the noise is actually improving vertex identification by introducing new vertices, not being identified in the smooth version. The reason this happens as we explained before, is due to the nature of the integral descriptors involved 


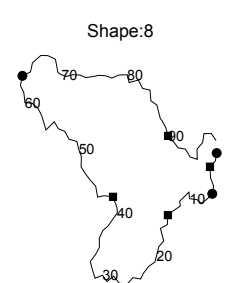

Shape: 9

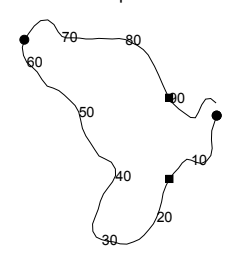

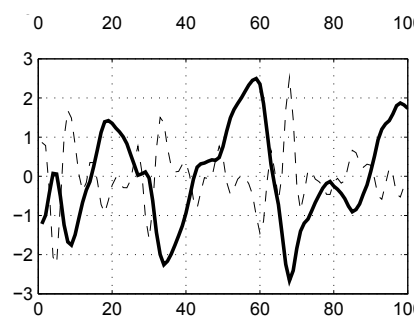

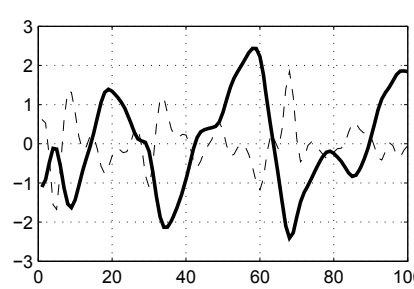

Fig. 7: Solid line is $\dot{\phi}$, dashed line is $\dddot{\phi}$. The method identifies undisputed points of extreme curvature (Global Vertices) on the shape's boundary in the co-localization of the above zero crossings. Local maxima curvature points (convex) are marked as solid circles, local minima (concave) are marked as solid squares. More points are correctly identified in the noisy version.

in the proposed method of localizing vertices. In a smoothed curve, a point of maximum curvature may or may not appear as extreme point on the boundary, depending on the location of the point with respect to the rest of the curve. Point No. 63 e.g. is not identified in the smoothed version since $\dot{\phi}$, even though it is close, it does not actually achieve zero crossing at a neighborhood of 63 . Applying noise in the neighborhood of 63 leads to greater diversity in the tangent directions around 63 and in the noisy version we see $\dot{\phi}$ finally achieving a zero crossing there and 63 correctly being identified as a vertex. This effect of noise is valid only for points that are close of being location extremes on the boundary and is therefore location dependent and has no effect for points that are not well located globally, e.g. point 15 where we see that, due to its specific location on the shape, no matter how much noise we apply to its neighborhood, it will never be identified as a vertex. In Figs. $(7,8,10)$, we also see how vertex identification is improved by inducing noise into various MPEG and KIMIA silhouettes.

\subsection{Comparison to LAII for Vertex Localization and Curvature Estimation}

A comparison to LAII [26] for estimating curvature but also for localizing vertices in noisy shapes is presented in this experiment. LAII is a low level descriptor of similar complexity to the proposed method, that generalizes the concept of curvature over the noisy segments of curves. A circle of certain radius is used, centered at each point, and the curvature is calculated as the ratio of the area of this circle that lies in the interior of the closed contour. 


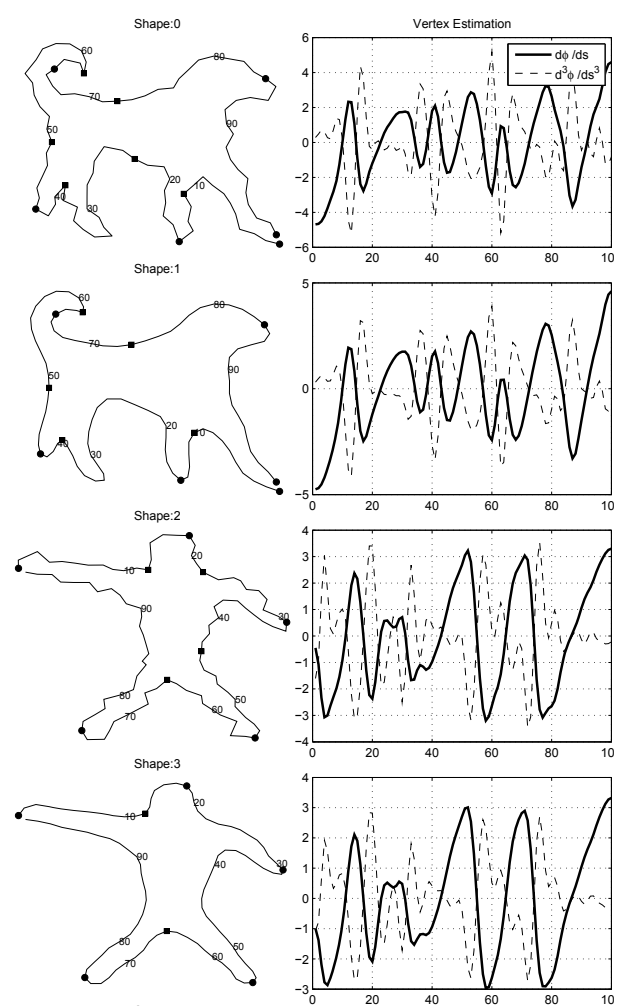

Fig. 8: Caption as in Fig. 7.

In the case of zero curvature, e.g. a noisy straight line, half of the disk will lie in the interior of the shape, whereas in the case of infinite curvature this portion will approach zero or to one depending on the sign of the curvature at this point. LAII therefore uses an integral (area of the circle) to estimate curvature but its essentially a local descriptor.

Our implementation of LAII is as follows: Starting from a binary image of the shape to be encoded, first we extract the boundary. The boundary is then discretized by sampling 100 equally spaced points on it.Then using a circular kernel (constructed as a binary image of a circle of radius 15, as is suggested in [26]) we convolve the filter with the shape image only at the boundary points. The values of the convolution at each of the boundary points are the values of the LAII estimated curvature at these points. For vertex localization we pick the LAII points of local minima and maxima, marked with stars and diamonds accordingly in Fig. (9) .

We compare this implementation of LAII to the proposed method of calculating curvature from equation (9), whereas vertex identification is performed as in the previous section by examining the co-localization of the local extrema of the total distance function $\phi$ with those of its second derivative $\ddot{\phi}$. 
Noisy Shape

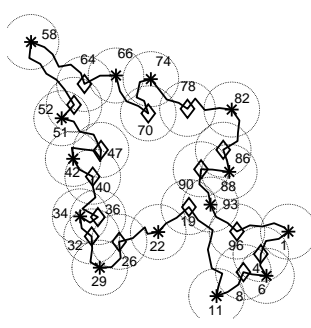

LAII Curvature Estimation

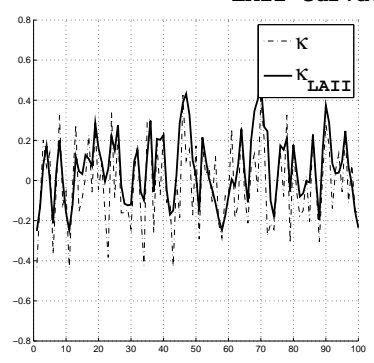

Smooth Version
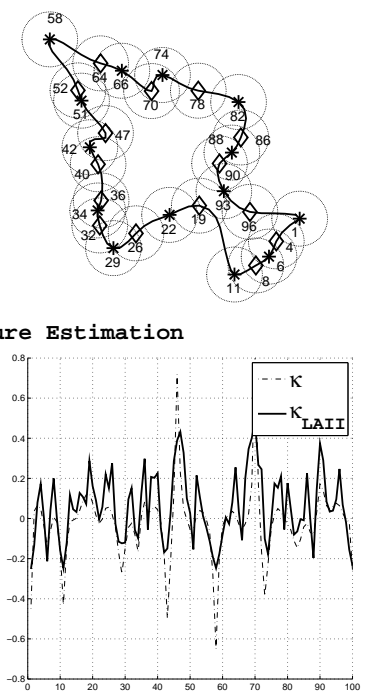

Fig. 9: Curvature estimation and vertex localization by means of the LAII method[26]. At the top row plots, stars and diamonds are curvature local minima and maxima respectively, identified by the LAII method from its curvature measurements, both for smooth and noisy versions of the same KIMIA silhouette. Compare this with the circles and squares identified by the proposed method in Fig. (6). At the bottom row of plots we see the differential curvature $(\kappa)$ and the curvature estimator according to $\operatorname{LAII}\left(\kappa_{L A I I}\right)$. LAII shows high resistance to noise, its curvature measurements are not affected by noise. However, the results in both curvature estimation and vertex localization are more fuzzy and less intuitive in comparison to the ones achieved by the proposed method in Fig.(6). The circles LAII uses around each identified point, are also plotted for easy visual verification.

For both methods the same extracted contours were used. Many advantages of the proposed method are apparent in this experiment. A comparison of the curvature estimators $\kappa_{\phi}$ and $\kappa_{L A I I}$ in Fig.(6) and Fig.(9) respectively, reveals better accuracy for the proposed method $\kappa_{\phi}$. The comparison is performed against the differential curvature which, as we see in the figures, collapses in the presence of noise. Also, the localization of vetices, marked in the top row of plots as filled squares and circles for the proposed method in Fig.(6), is more accurate and intuitive than the respective stars and diamonds marked as such by the LAII method in Fig.(9). In both the smooth and noisy versions the LAII localization of vertices is often mislead by local boundary formations. The middle row plots in Fig.(6) reveal the co-localization mechanism of the proposed method. 

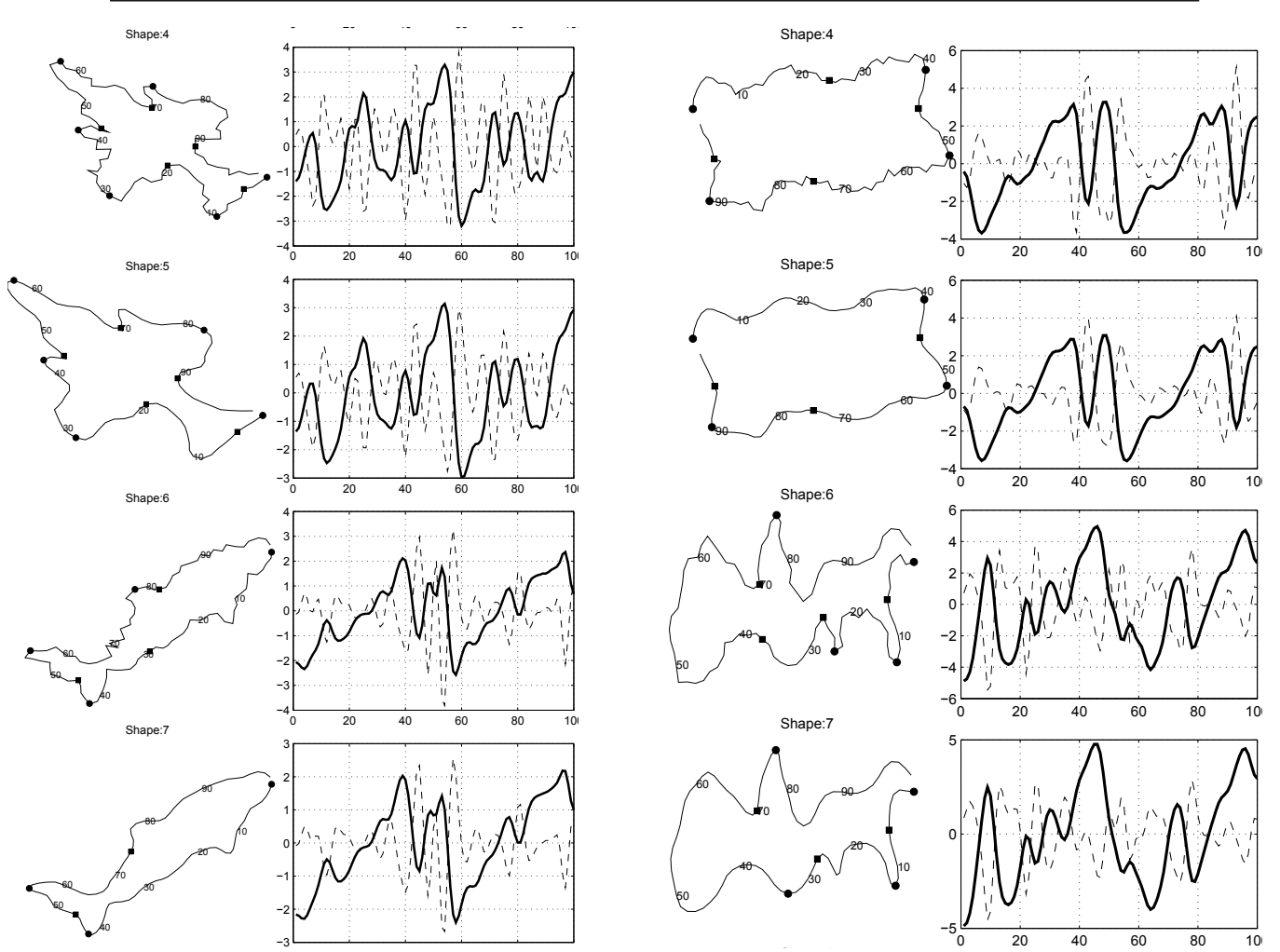

Fig. 10: Caption as in Fig. 7.

\section{Experimental Investigation on Incremental Noising with Deterministic Recursion}

Here we extend the method of noising to that of incremental noising by inducing deterministic perturbations on the boundary in a recursive fashion and we will examine how well VAR-based identification of vertex points performs in this case, in relation to various local methods at different degrees of locality. This new noising process can be performed in an additive manner to the existing boundary, also not affecting the initial boundary points. In the discrete case of a digital curve, for each pair of consecutive points on the initial boundary, a new point is added at the intersection of the circles centered at the original points and having equal radii of a certain length, greater than half the distance between the two original points. The difference with the noising defined in the previous section is that there the radii of the circles were drawn from a Gaussian distribution, whereas here the radii are constant as a parameter of the method. Furthermore here, noising is applied recursively, doubling the curve's points at each successive step. This incremental noising, proposed here, is investigated for the first time in its combination with VAR for vertex identification. Different implementations involving the locations of $\dot{\phi}$ and $\phi$ 
zero-crossings are compared against local, localized and smoothing methods that employ various degrees of locality.

Incremental noising works, because the use of VAR-based global representation of vertices, turns the negative local effects of noise into positive effects within the global analysis framework, as the preceding theoretical analysis demonstrated. For the proposed method in particular, noising is an enabler. Vertices are detected directly without the need for curvature calculations. Recursively applying noising in the proposed manner forms neighborhoods of increasing differential order around the initial curve points, resulting in a concept that is analogous to that of progressive smoothing. Thus incremental noising for global methods, can be viewed as a conceptual duality to what progressive smoothing is for local methods. In the next sections we further investigate these concepts.

The baseline methods designed for comparison are derived from the different ways one can define discrete curvature on a digital curve, according to the fundamentals in the literature. The implementation is our own, because the methods should be as clear and as simple as possible, so the reader can focus on the scope of locality employed by each method, and how incremental noising is affecting them. The experimental results on the effects of incremental noising on vertex localization are better understood this way. The baselines are meant to serve different definitions (implementations) of curvature so as to see how incremental noising is affecting the task of vertex localization under these different implementations.

A surprising result is revealed in the experiments. The proposed VARbased global method with incremental noising, identifies on distorted shapes, the same points that progressive smoothing identifies on the corresponding undistorted ones. Deterministic incremental noising in other words, seems to have the effect of gradually removing Gaussian distortions on the boundary, in terms of identifying vertices on the corresponding smooth shapes. This result is counterintuitive and was reached by means of precision vs recall (PR) comparisons in identifying vertex points (vertices) on the boundary of the KIMIA[27] benchmark dataset of silhouettes for various local, localized and smoothing methods that assume different degrees of locality in their treatment of noise. We offer a unifying explanation of this connection between incremental noising and progressive smoothing.

\subsection{Experimental Design}

The KIMIA dataset consists of 9 classes of 11 shapes each and has been used expensively in the literature to benchmark the performance of various classification algorithms. Here we use shapes from all classes, to produce representative noisy versions of shapes from each class and test various methods on their ability to identify Vertex Points (VPs) on their noisy boundaries. Term noisy is used to describe the resulting contours after Gaussian noise has been induced on the original contours as in the previous experiments in section 
8. The resulting noisy contours are further processed here with noising. The term noising is used to describe the process of making a contour of $P$ points $P>100$ from a contour of $P / 2$ points after adding new points to it in a principled manner as was explained in section 9. From each noisy contour of $n$ points a noising step produces another noisy contour of $2 n$ points. Similarly in a recursive manner contours of points $2^{k} n$ are produced for each KIMIA silhouette, $k$ being the $k^{\text {th }}$ noising step for $k=1, \ldots, 4$. Precision vs recall (PR) measurements, of the proposed method against local and localized variants of methods for identifying vertex points on the boundary of the above noisy versions of shapes are appropriately designed and performed in the following sections.

\subsection{Ground Truth}

We define the ground truth vertex points (GT-VPs) as the set of points where the cumulative curvature function achieves local maximum or local minimum. The cumulative curvature for a certain shape is defined as the point-wise addition of the curvatures of its progressively smoothed versions. In Fig.11 the cumulative curvature function is shown together with the progressive smoothed versions of KIMIA silhouettes from all classes. The vertex points detected at each smoothing step are also shown as the local extrema of the respective curvature functions (vertices). GT Vertex Points (VPs) are calculated as in Fig.11 only for the original KIMIA silhouettes of 100 points and they are assumed to their respective locations when the number of contour points are changed with noising. If e.g. point No $n, 1 \leq n \leq 100$ was designated as a GT VP on the original 100 silhouette contour, then point No $2^{p}(n-1)+1$, where $p=\log _{2}(N / 100)$, is also a GT VP of the noisy contour of $N$ points, $N=100,200,400,800,1600$. This algorithm of defining GT VPs is denoted by $S K$ (from Sum of Curvatures (K)) and is included in the experiments as the method one would expect to be the most successful in tracking back these GT points from the noisy versions. As we will discuss later however, the proposed method achieves better Precision/Recall (PR) performance in tracking back these points, even though $S K$ is the method that defines them in the first place.

\subsection{Methods Under Comparison}

Colors and markers below refer to Fig.(13).

- Local Area Integral Invariant (AI): Using a circular kernel (constructed as a binary image of a circle of radius 15, as is suggested in [26]) we convolve the filter with the shape image only at the boundary points. The values of the convolution at each of the boundary points are the values of the $A I$ estimated curvature at these points. As VP we pick the points 

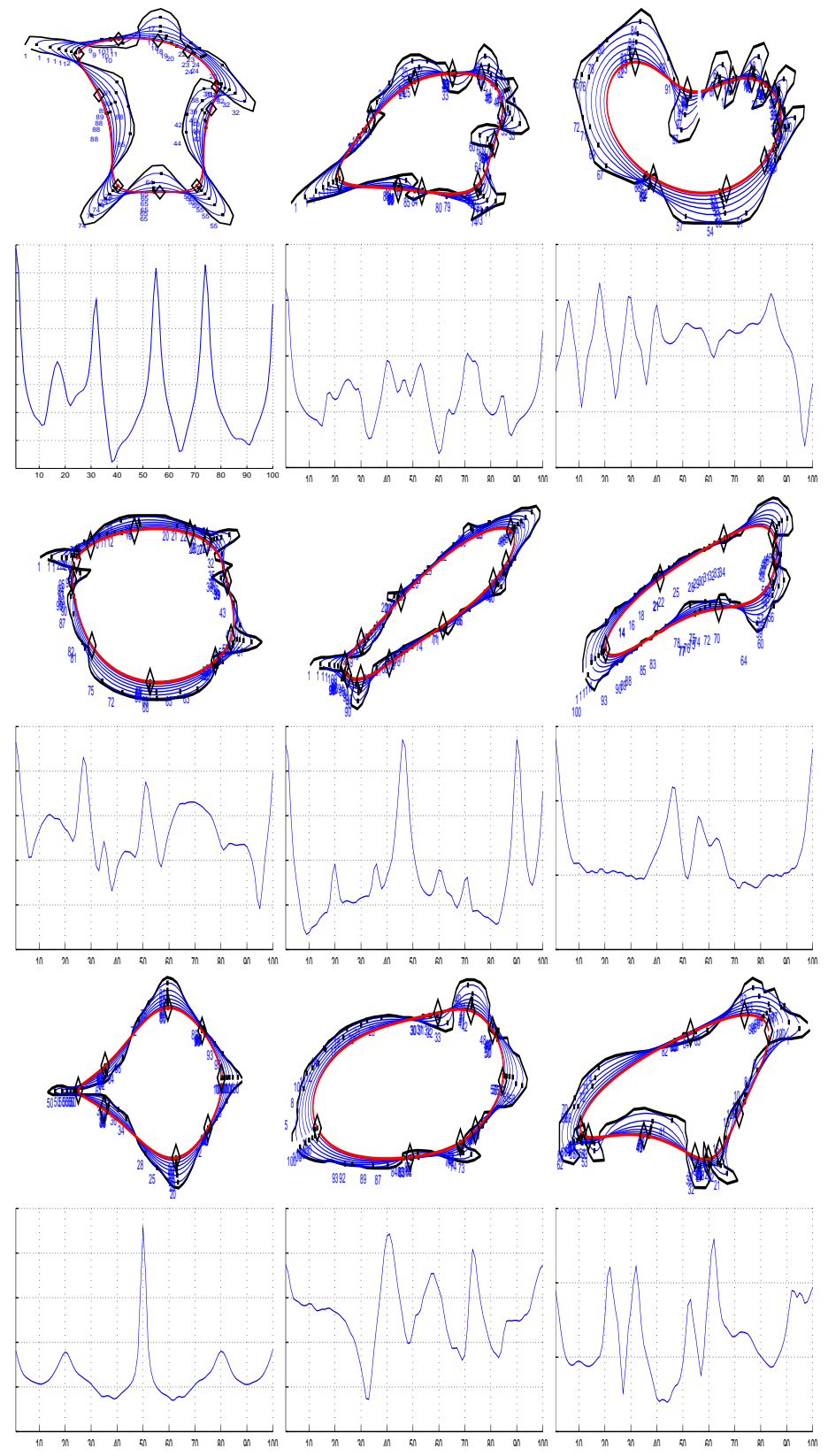

Fig. 11: Calculation of the ground truth (GT) set of Vertex Points (VP) of representative KIMIA silhouettes from all classes. The initial curve is shown with the thick black outermost contour, the final curve is shown as the red innermost contour. Intermediate curves produced by progressively smoothing the initial curve are shown as blue contours. Solid squares are tracking the local extrema of curvature at each smoothing step. The final ground truth points are shown as black diamonds. These are the local extrema of the cumulative curvature shown at the respective lower halfs. 
where the $A I$ descriptor attains a local minimum or local maximum. Complexity: $O\left(k^{2} \times n\right)=O(n), k$ being the size of the kernel and $n$ the number of contour points + at least one order if dynamic adjustment of the kernel size is needed in noisy conditions. Parameters: Circle Radius: 15, Color: red, Marker: star.

- Proposed, 1st implementaion $\left(V_{o}\right)$ : In the first GL equation (7) the first derivative of $\varphi$ is calculated as an integral over parametric angles, no distances are computed either and there is no derivative involved. For the third derivative of $\varphi$, a similar calculation is not apparent from the theory. For this reason we decided to provide two implementations of the proposed method. The first, signified by $V_{o}$ uses the zero crossings of $\dot{\varphi}$ only and identifies VPs there without calculating derivatives. Complexity: $O\left(n^{2}\right)$ regardless of noise. Color: blue, Marker: Circle.

- Proposed, $2^{\text {nd }}$ implementation $(V)$ : The second implementation signified by $V$ uses the collocation of the first and third derivatives. For the calculation of $\dddot{\varphi}$ see the relevant section 9.5. Complexity: $O\left(n^{2}\right)$ regardless of noise. Color: Green, Marker Cross.

- Heron Curvature $(K)$ : For each contour point $p_{i}$ a triangle is defined having vertices $p_{i}, p_{i-k}, p_{i+k}$. Heron Curvature at $p_{i}$ is defined as the area of this triangle. VPs are considered the local extrema of Heron Curvature. Complexity: $O(n)$ regardless of noise. Color cyan, Marker none.

- Cumulative Curvature $(S K)$ : Same with cumulative curvature for defining GT VPs above (section 9.2) with the only difference that $S K$ will run on the noisy contours. Complexity: $O\left(n^{2}\right)$ regardless of noise. The number of smoothings for calculating the cumulative curvature are a function of the number of points. Color magenta, Marker square.

The methods under comparison were chosen to be pure approaches that emphasize different degrees of locality in solving the problem of VP detection, thus the investigation could remain focused on the essential concepts (e.g. what degree of locality is involved in the concept of an VP under the presence of noise?), rather than dragged into heuristic implementations directed to specific datasets or specialized problems. $K$ is a local method binded to a 1D locality, defined as a portion of the contour length, $A I$ binds to the locality of a $2 \mathrm{D}$ disk of a certain radius, whereas the proposed method is globally defined $(\dot{\varphi}$ defined through an integral) and finally $S K$ is a hybrid method since both the vertices (local) but also the tracking of them across scales (global) are used by the method.

\subsection{Performance Metrics}

Every shape will in general have different number of ground truth (GT) points and each method under comparison will in general identify a different number of Vertex Points (VP) for such each shape. The challenge therefore in designing a strategy for comparison is in defining precision and recall in such a way that will not bias in favor of a particular method. We can hardly do better than 
using a standard probabilistic framework to calculate probability densities of a point being an VP under a particular method. For each method and for each point on the boundary we calculate the probability of this point to be an VP under this method as the reciprocal of its boundary distance to the nearest VP point predicted by this method. Here we imply a uniform distribution which is a plausible and unbiased assumption. Suppose therefore that $L_{m}=\left\{l_{i}\right\}, i=1, \ldots, n$ is the set of the $n$ VPs predicted by method $m$. For each point $p_{j}$ on the shape's boundary the probability of $p_{j}$ being an VP under method $m$ according to the uniform distribution is:

$$
\operatorname{pr}\left(p_{j} \in V P\right)=d s\left(\min _{i}\left(\left|p_{j}-l_{i}\right|\right)\right)^{-1}
$$

where $d s$ the arc element. This is indeed so since $\left(\min _{i}\left(\left|p_{j}-l_{i}\right|\right)\right)^{-1}$ is the probability density for the uniform distribution in the interval $\left(p_{j}, l_{i}\right)$ if $l_{i}$ is the closest VP to $p_{j}$, identified by method $m$. The probability in other words, of a noisy point $p_{j}$ (point on a noisy version of the curve) being an VP at the original smooth version of the same curve according to a particular method, is inversely proportional to its distance to the nearest of the VPs identified by this method on the noise curve. As is typical with probability densities we assume $l_{i} \neq p_{j}$, their distance in other words is never zero but achieves a minimum value.

Each method under comparison therefore, defines a probability distribution over points on a noisy curve that measures their potential to be the VPs of the original curve. But since the actual VPs are provided in the set of ground truth (GT) points as above, the methods will be compared on the cumulative probability they assign to the points that lie at the same location with the GT points.

A concern when designing the experiment was that local methods under noisy conditions typically identify VPs everywhere, densely at many locations, therefore VPs are randomly detected close to GT points. The probability framework just introduced compensates for this case since normalizing the probability density will distribute the probability mass evenly among all the points with low values at each point. When on the other hand, a method identifies fewer VPs, normalizing the probability density will produce picks of high probability mass at these points and low probability mass at the other points. Identifying a correct location close to GTs is thus more critical in the latter cases. The probabilistic framework therefore is consistent with our intuition that a trade-off should exist between the number and the importance of location of the points identified by each method.

PR graphs are usually considered in a discrete context to measure the quality of matching in benchmarked classification tasks [28]. If a method, in order to get a correct match on the $m$ th GT item, has previously matched correctly only $n, n \leq m$ items, then the respective PR graph holds at the recall position $m$, ( $m$ the items that have been recalled so far), the value $\frac{n}{m}$, the ratio of the correct items over all items that were classified so far. In our experiment such a discrete approach was not apparent since there are different 

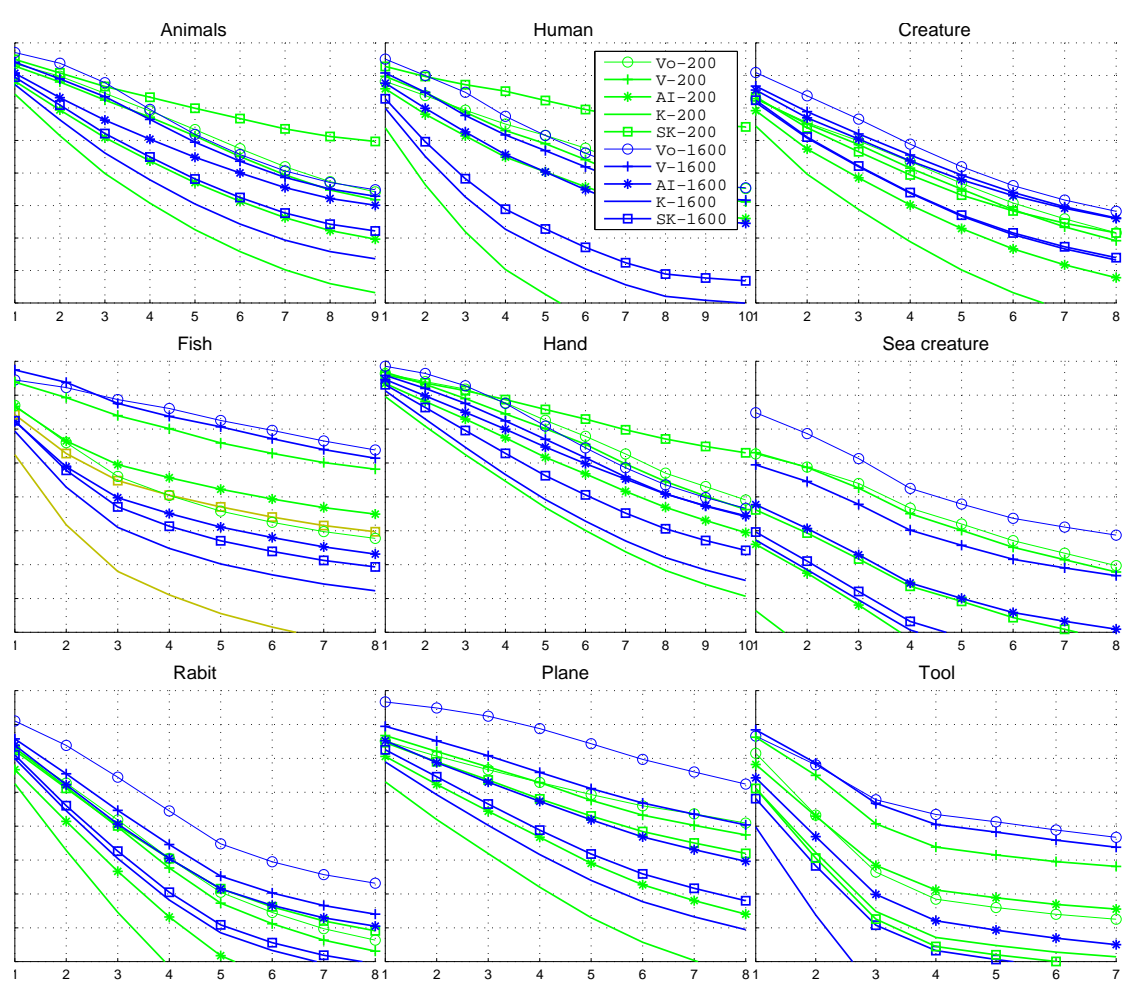

Fig. 12: Precision-Recall diagrams for each class of the KIMIA benchmark dataset of silhouettes. Blue graphs correspond to 1600 point contours, green graphs to 200 point contours. The PR graphs of all the 11 shapes of each class where averaged and presented here for each noising scenario. Horizontal axis is indexed with the VPs for each shape, vertical axis holds the precision in the range $[0,1]$ but is omitted for better display. See section 9.6 for a discussion on the results.

GT points to be recalled by methods that produce a different number of VPs. However, the probabilistic approach just introduced, can be seen as extending the discrete PR concept above, to continuous values, where instead of hit or no hit we have the degree of a hit, measured by the diversion of the method's density from the GT density at the location of the GT points.

Since each method produces a probability density over all the points on the curve, this density can be compared to the GT density, produced by the GT method also over all curve points. This way, a common reference was made possible. The PR graph therefore, holds at the recall position $m$ (number of GT points recalled so far) the value $1-\sum_{i=1, \ldots m}\left|p(i)-p_{G}(i)\right|$, where $p(i)$ and $p_{G}(i)$ are the method's density and the GT density respectively evaluated at the GT point $i$, and $\sum_{i=1, \ldots m}\left|p(i)-p_{G}(i)\right|$ is the cumulative absolute diversion this method's density has from the GT density at the points recalled so far. We subtract from 1 to be visually consistent with the usual image of a PR 
graph that drops with precision. For each density, the absolute differences of its values from the GT density values at the GT locations are sorted in ascending order and subtracted from 1 to be consistent with the Precision-Recall (PR) formulation. The final PR graph is a cumulative progressive addition of these sorted values. A perfect match will produce zero differences from the GT density at the GT locations (the method's density will be identical to the GT density in this case), therefore the visualized Precision Recall graph will be a constant 1 at all the GT locations. The best matches (smaller absolute differences from the GT density values) are sorted first. The PR graph drops as more GT VPs are examined and more errors are accumulated.

\subsection{Implementation Details}

Local extrema and zero crossings are calculated for scalar descriptors using a level set approach. A sliding window (1D window as a portion of the boundary in the form of $[s-d s, s+d s]$, where $s$ contour length parameter) is used on the values of the descriptor. The values left and right of the window center are subtracted from the center value in pairs and a local extreme is identified if all these differences have pairwise the same sign. The size of this window is the same for all methods and equals 0.017 as a ratio of the total contour length, therefore is invariant to the number of contour points. All local extrema calculations for all methods under comparison are calculated in this way using the same window size ratio. The experiment is now described in steps:

1. Each silhouette in the KIMIA dataset is discretized by 100 equally spaced points and the ground truth GT set of VPs is calculated.

2. A 100-point noisy version of each silhouette is constructed. Gaussian noise is applied by moving each point on the direction of its normal by a random quantity drawn by the normal distribution with variance 2.0.

3. Noising is applied on each of the 99 100-points noisy silhouettes of the previous step producing 99 200-points noisy silhouettes. Similarly contours of 400, 800 and 1600 points are produced as explained in section 9.1. Each noising step adds a new point at the middle of each edge with a constant normal perturbation equal to 0.01 times the magnitude of that edge.

4. The 5 methods of 9.3 are used to compute vertex points on each of the noisy silhouettes of the previous step. In method $V$, for the existence of the $\dddot{\varphi}$ zero crossing a level set approach is applied: Since the locations of the local extrema of $\varphi$ have been identified on the zero crossings of $\dot{\varphi}$, one can infer the behavior of the third derivative of $\varphi$ by examining the shape of $\varphi$ around those points following a level set approach (since $\varphi$ is a scalar). A zero crossing of the third derivative in the vicinity of these zero crossings means a local extreme for the second derivative of $\varphi$ and thus a sudden change (above a threshold) in the values of $\varphi$ around its local extreme. The same sliding window strategy of the same size as above is used but now at least one of the pairwise absolute differences from the center must also be greater than 0.15 times the window length. $S K$ is the only method 
that smooths the curve and it does so progressively, it is thus the same method that calculates the GT set initially, but now works on the noisy curves after noising.

5. For each noisy contour (after noising) and for each method, the probability densities are estimated according the section 9.4 based on the VP set predicted for each contour by each method.

6. The comparison of the various densities (methods) is performed against the GT density as explained in section 9.4.

The execution time for one contour of 100 points was less than a second on a computer with standard configuration for all methods. All methods had similar performance as contour points increased. The worst performance was observed in relation to the $S K$ method since multiple smoothings increased computational time in contours that exceeded 1600 points.

\subsection{Results}

In Fig.(12) PR measurements are presented per KIMIA shape class for all methods and for two noising scenarios. Green curves correspond to shapes affected by a 2 step noising (200-points noisy shapes) while blue curves correspond to a 5 step noising scenario (1600-points noisy shapes). See legend for the markers used for each particular method. In Fig. (13) details of the process is shown for characteristic shapes of 2 KIMIA classes. See caption for details on the presentation. Since performance is judged against GT VPs identified by the $S K$ method one would naturally expect $S K$ to perform extremely well in this set of experiments. We see in both of the above figures that $S K$ is affected by noising although it still performs well especially for shapes that have many dominant VPs of positive and negative curvatures. Even in those cases however, noising improves the proposed method $V_{o}$, especially for the most dominate vertex points (best matches).

The proposed $V_{o}$ is better than all the other methods, the superiority gap, seen as the vertical distance of PR curves, increases with noising. Compared to $S K, V_{o}$, behaves better in most shape classes. For certain classes of shapes with many dominant points of positive and negative curvatures (Hands, Humans, Animals) $S K$ identifies GT VPs more accurately for low noising scenarios but as noising increases the performance of $S K$ deteriorates, while $V_{o}$ improves to a point where it outperforms $S K$ for the best 2-3 vertex points. It is important to notice for one more time that $S K$ is a biased estimator in this experiment.

We also notice that both implementations of the proposed method improve with noising. As noising increases the proposed method identifies less points in contrast to the other methods where more points are identified as noising is recursively applied. This improvement at the PR graphs is particularly apparent for the first 3,4 best vertex points, and this is easily explainable since less but more accurate points are correctly identified after noising. This behavior can be observed e.g. in Fig.(13) airplane class, where the PR graph improves with noising to an almost perfect score for the best 5 hits at the 4 th 


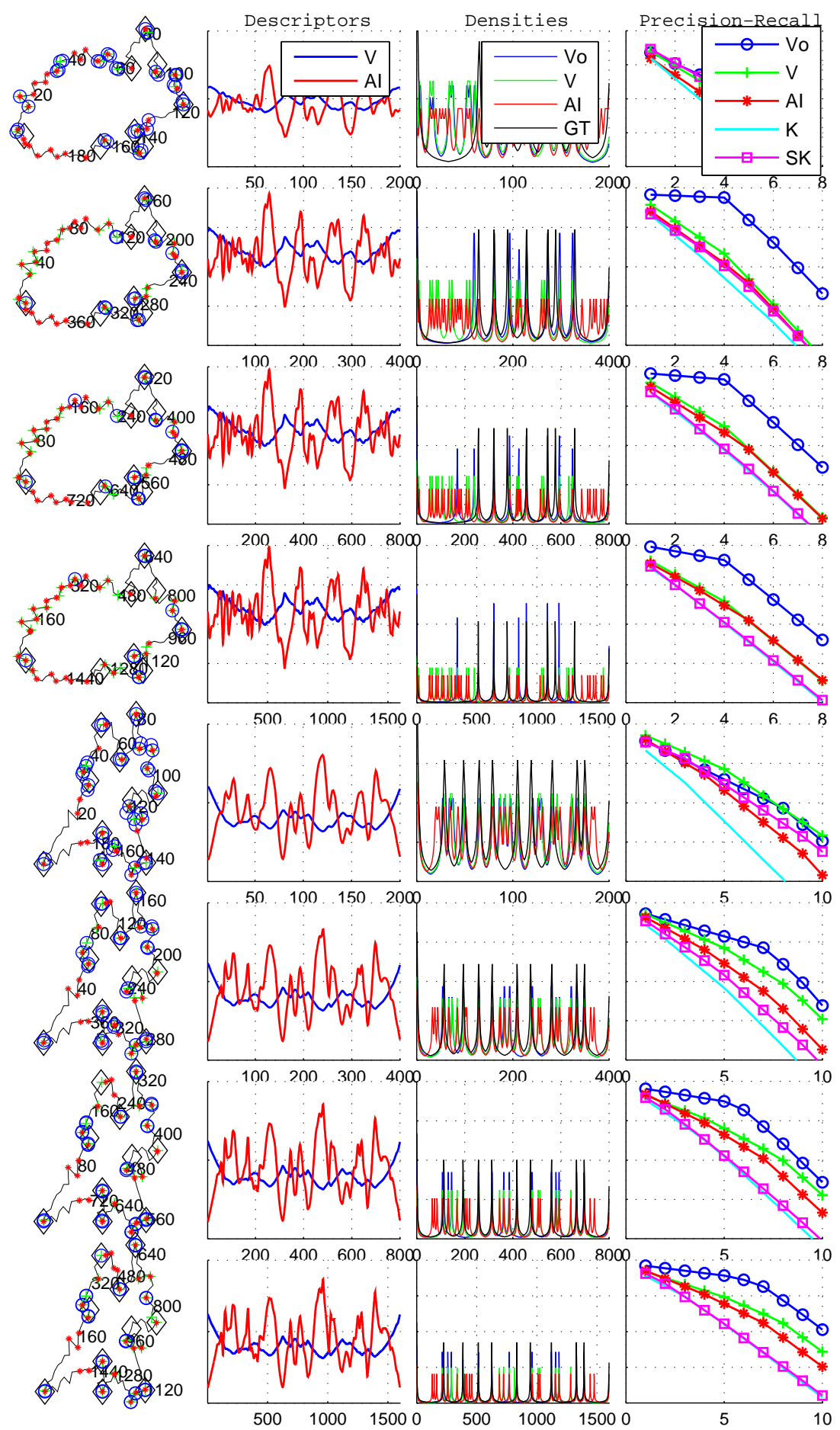

Fig. 13: $1^{\text {st }}$ column: Noisy versions of KIMIA shapes from representative classes in quartets of increasing noising of 200, 400, 800 and 1600 points in vertical order. Big black diamonds represent the Ground Truth (GT) points identified by the cumulative curvature method as in Fig.(11) on the corresponding smooth silhouettes of 100 points. The positions of the GT points on the noisy shapes were extrapolated from those of the smooth shapes. GT points were calculated only for the initial KIMIA silhouettes of 100 points. Blue circles, green crosses and red stars represent Vertex Points (VPs) identified by $V_{o}, V$ and $A I$ methods respectively. $2^{\text {nd }}$ column: $A I$ and $\operatorname{VAR}(\varphi)$ descriptors, where the x-axis is marked by the number of contour points and the $y$-axis has been omitted for better display, since only the locations of local extrema are important in this graph. $3^{\mathrm{d}}$ column: Probability densities corresponding to $V_{o}, V, A I$ and Ground Truth $G T$ descriptors where the x-axis is marked by the number of contour points. The y-axis range is $[0,1]$ but was omitted for better display. $4^{\text {th }}$ column: Precision-Recall $(\mathrm{PR})$ graphs for the 5 methods under comparison where the $\mathrm{x}$-axis marks the GT points (the cardinality may differ among shapes) and the $\mathrm{y}$-axis range is $[0,1]$ but was omitted for better display. The PR graph for a method, accumulates the diversion this method's density has from the GT probability density as more GT points are recalled. See Section 9.6 for a discussion on the results and Fig.12 for per class average PR graphs. 


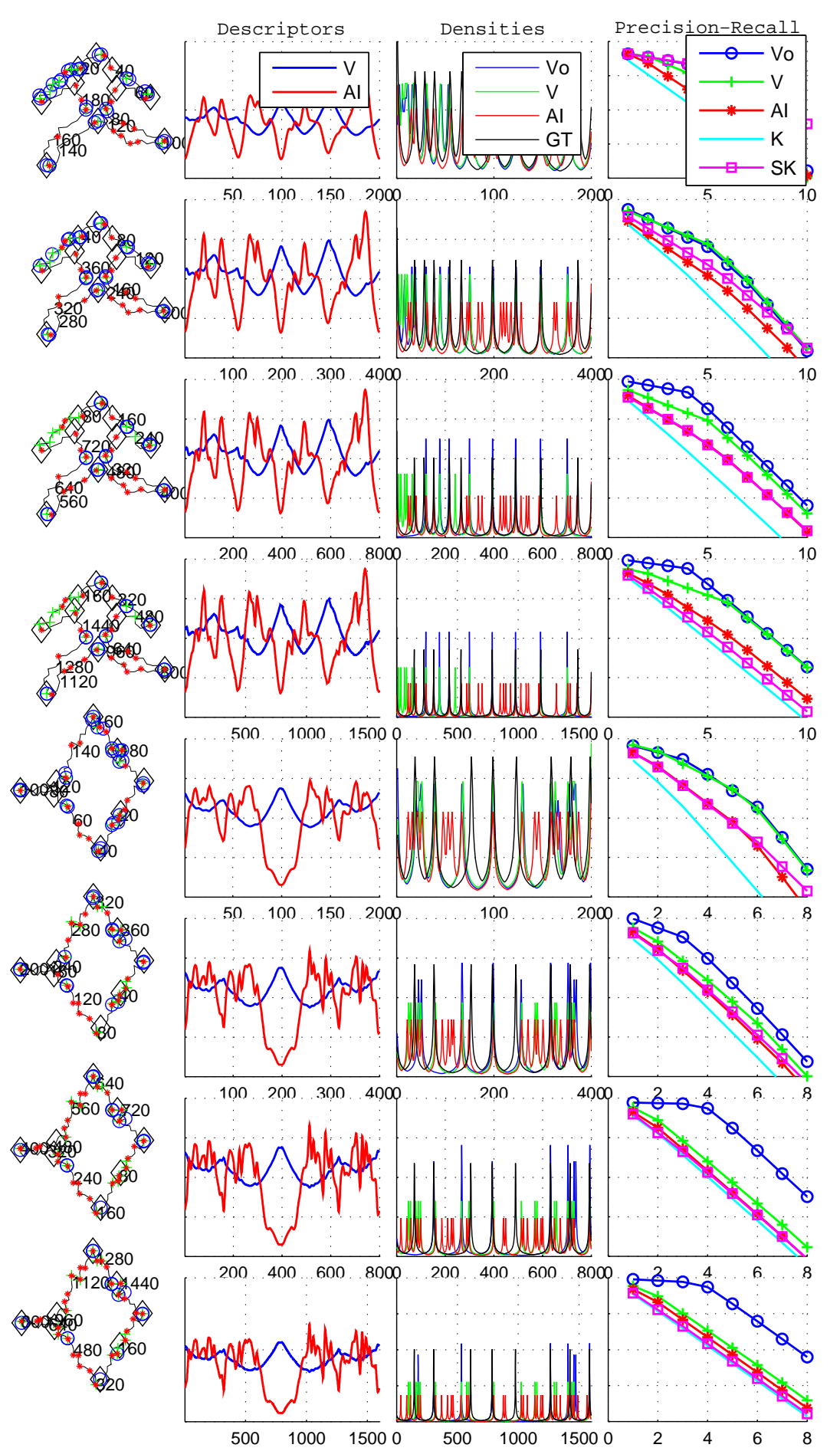

Fig. 14: Caption as in Fig.13 above. 


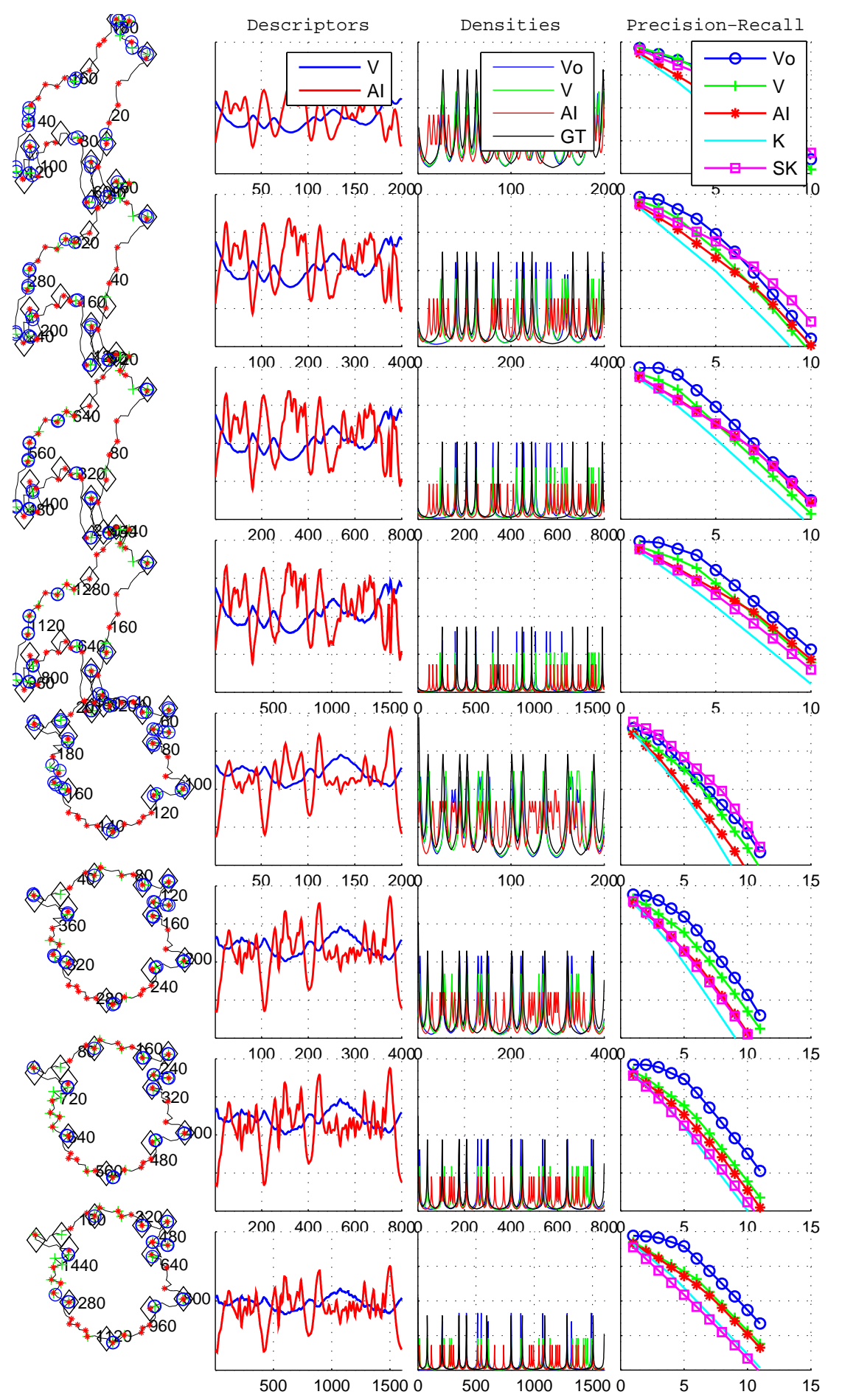

Fig. 15: Caption as in Fig.13 above. 


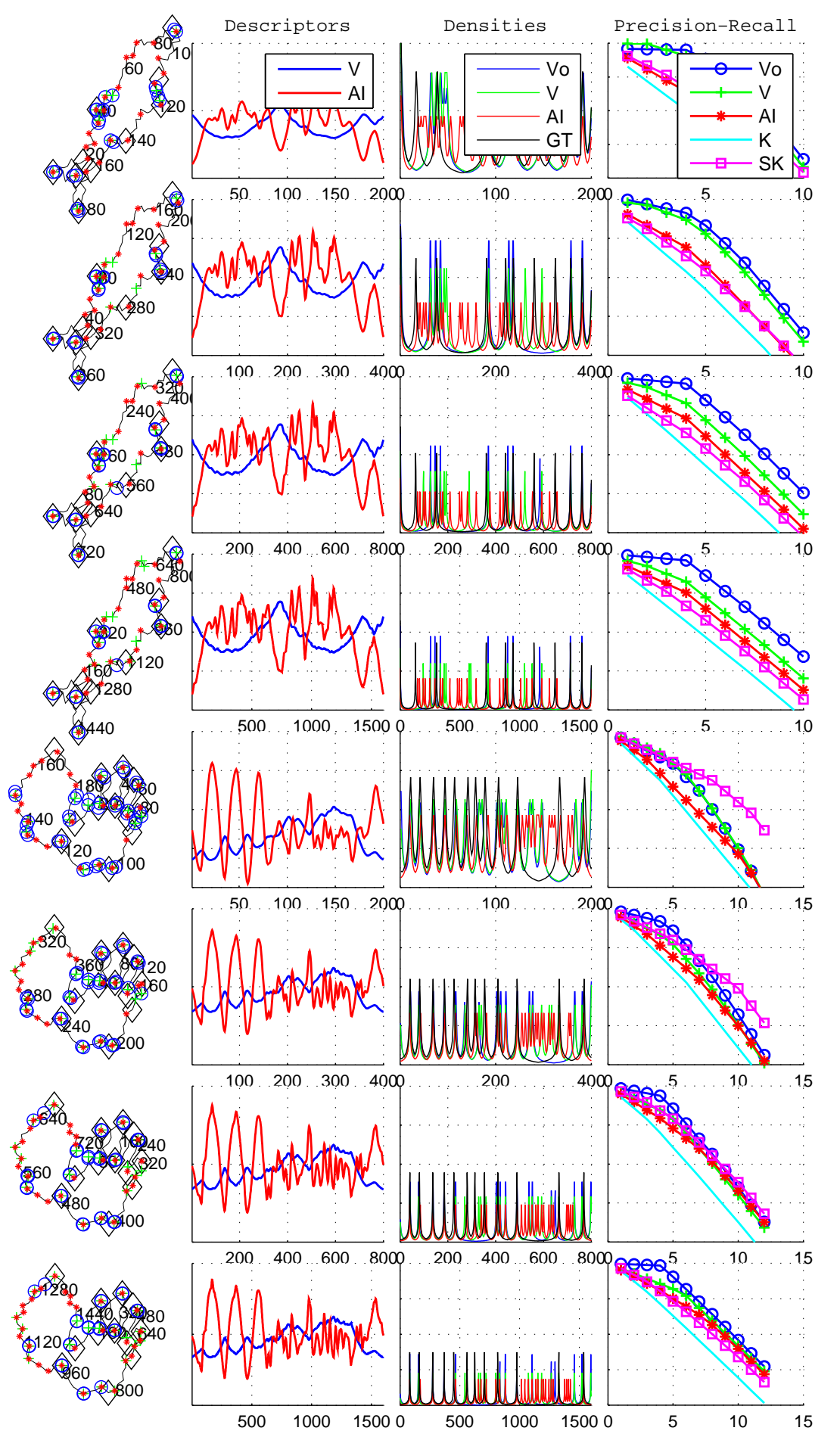

Fig. 16: Caption as in Fig.13 above. 


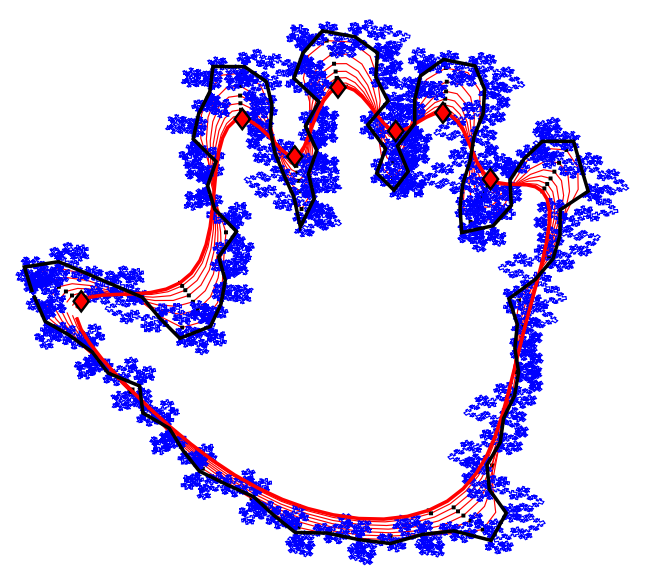

Fig. 17: Space filling properties of incremental noising and progressive smoothing. The black curve is the initial contour, red curves are the progressive smoothing versions of the initial contour, thick red line is the final smoothed version of the initial contour, red diamonds are the persistent vertices identified by both methods. The blue curve is the initial curve after 10 levels of noising $\left(2^{10} 100\right.$-points contour) filling the space around the initial contour in a fractal manner. Both progressive smoothing and incremental noising detect persistent vertices at places of increased $2 \mathrm{D}$-space coverage.

noising step (1600points), while the score at the $2 \mathrm{~d}$ noising step (200 points) was comparable to the other methods. Similar behavior, where the best 4-5 hits approach a perfect score with noising can be observed for the rest of the classes of the same figure, and eventually for all the KIMIA classes from Fig. (12) where the scores from all shapes per class are combined.

\subsection{Discussion}

In the experiment above, a surprising result is obtained. On distorted shapes, incremental noising performs better in identifying Ground Truth (GT) points that were defined by progressive smoothing on the corresponding undistorted shapes. This can happen only if: (a) The two methods are effectively identifying the same vertices, and (b) incremental noising is more robust to Gaussian shape distortions than progressive smoothing. Even though the two methods are conceptually orthogonal there must be a link that connects them in the context of vertex identification. In this direction, one may notice that the shape features that are persistent in the course of progressive smoothing are 
also the ones that are emphasized by the space filling properties of incremental noising.

Indeed, the successive contours produced by progressive smoothing fill up the 2D space between the initial contour and the final smooth version. Persistent vertices are identified in places where the intermediate contours shrink faster per single smoothing, thus the local space coverage is increased. Incremental noising on the other hand, adds more points to the initial contour at each step, producing a curve that covers 2D space around the original contour in a fractal manner (blue colored space filling curve in Fig.(17)). As one can see in the same figure, persistent vertices are located in areas where the 2D-space filling curve is more dense and these are the same areas where progressively smoothed contours cover more ground as well. The fractal curve produced due to incremental noising will fill up more area around strong vertices than it will around weaker vertices and this is why stronger vertices persist as well with noising as they do with smoothing. Incremental noising works because it is used in combination with VAR's global characteristics. As has been already explained, VAR is sensitive to relative location on the curve and less to noiselike curvature. The fractal curve produced by incremental noising evolves in a way that eliminates location specific characteristics faster around weak vertices than around stronger ones. In smooth shapes therefore, the two methods identify the same points, after distorting the shapes however, incremental noising seems more robust in tracking back those same points, exhibiting a noise removal behavior in that sense. But this is not the only advantage of incremental noising in this task. Smoothing is a lossy operation whereas noising is not as only new points are always added to the previous ones. A sub-sampling can always produce the initial curve. Furthermore, the space filling coverage due to incremental noising converges to a certain area around the original contour that depends on the initial noising parameter. Progressive smoothing on the other hand always covers the same area, namely the one between the initial contour and the final smooth version, the space covering behavior is thus more discriminative and better controlled in the case of incremental noising.

\section{Conclusion}

The proposed method is an attempt to bring in perceptual characteristics to the low level task of vertex identification by combining a robust vertex estimator $\ddot{\phi}$ and a global position estimator $\phi$. The non trivial local extrema of the former are identified in the zero crossings of $\dddot{\phi}$ and is already a robust vertex estimator, since it is based on the distance to the rest of the curve and not on local to the curve differentials. In the experiments and also according to the theory in the manuscript, one could follow that $\ddot{\phi}$ can be used as a vertex estimator by itself and vertices could be defined directly on its local extrema (zero crossings of $\ddot{\phi}$ ) accordingly. However, although robust in comparison to the differential alternatives, vertices identified this way would be according 
to the formal mathematical definition, lacking any attempt of differentiation with respect to their importance. This is where $\phi$ plays an important role.

The introduction of $\phi$ in the process of identifying vertices, provides an additional capability, that of acquiring a global view of the locations of the vertex points, introducing the concept of Global Vertices, as undisputed points of extreme curvature that due to their location at the extremes of the shape (zero crossings of $\dot{\phi}$ ) are characterized as perceptually important ${ }^{3}$. The key concept behind Global Vertices is that extreme location implies extreme curvature. Extreme points on the curve cannot exist without extreme curvature there. The inverse does not hold for all vertices, thus the distinction between Global (according to Proposition 3) and regular (according to the traditional differential definition) vertices.

One can thus say that the co-location of the zero crossings of $\dddot{\phi}$ and $\dot{\phi}$, can be interpreted as combining extreme curvature with extreme location, thus an attempt to introduce global perceptual characteristics to a problem that is inherently of a local nature. In the experiments we have used characteristic shapes from the KIMIA and the MPEG datasets in pairs of smooth and noisy versions. Notice that while $\ddot{\phi}$ zero crossings alone could identify regular vertices, the co-location with $\dot{\phi}$ zero crossings results in picking only the Global vertices, the undisputed points of extreme location, in an attempt to introduce perceptual meaningfulness in the process. Since extreme location is a global feature, adding noise to the shape has no significant effect. In fact, the only result is to introduce a few more extreme points that were very close, but didn't quite appear as such in the smooth version and this is the mechanism behind improving the results by noising.

One could also notice here that $\ddot{\phi}$ is used as a vertex estimator and not as a curvature estimator, since curvature is estimated by equation (9) in the manuscript, thus the herein presented method calculates vertices directly, bypasses curvature and brings noise on our side. As is shown in the experiments, there are cases of shapes where different noisy versions favor certain vertices. For this reason, the method in practice runs for various noisy scenarios finally considering the union of the vertices identified in each of the scenarios. Furthermore, one could control the accuracy of zero crossings co-localization by adjusting the width of the finitesimal interval in which a co-localization is assumed to have occurred, achieving this way different degrees of location dependence. Further tuning of the method can be achieved by posing restrictions on the slope of the zero crossings or the magnitude of $\ddot{\phi}$, resulting in further meaningful differentiation among vertices.

\section{References}

1. K. Raftopoulos, S. Kollias, Computer Vision and Image Understanding 115(8), 1170 (2011)

\footnotetext{
3 The fact that remote points are perceptually important has been demonstrated in [1] where shape matching scores in benchmark datasets where improved by permitting correspondences only to remote points.
} 
2. J.R. Bennett, J.S. Mac Donald, IEEE Trans. Comput. 24(8), 803 (1975)

3. P. Rosin, J. Zunic, in Handbook of Applied Algorithms: Solving Scientific, Engineering, and Practical Problems, ed. by A. Nayak, I. Stojmenovic (John Wiley and Sons, 2008), pp. $347-372$

4. J. Flusser, B. Zitova, T. Suk, Pattern Recognition 15(12), 3784 (2006)

5. D. Xu, H. Li, Pattern Recognition (PR) 41(1), 240 (2008)

6. J.D. Zunic, P.L. Rosin, L. Kopanja, IEEE Transactions on Image Processing 15(11), 3478 (2006)

7. M. Stojmenovic, J. Zunic, JMIV 30(1), 73 (2008)

8. J.D. Zunic, K. Hirota, P.L. Rosin, Pattern Recognition 43(1), 47 (2010)

9. S. Abbasi, F. Mokhtarian, J. Kittler, Multimedia Syst. 7(6), 467 (1999)

10. D. Zhang, G. Lu, J. Visual Communication and Image Representation 14(1), 39 (2003)

11. S. Belongie, J. Malik, J. Puzicha, Pattern Analysis and Machine Intelligence, IEEE Transactions on 24(4), 509 (2002)

12. N. Sladoje, J. Lindblad, IEEE Transaction on Pattern Analysis and Machine Intelligence 31:2, s. 357-363 (2009)

13. S. Biasotti, L.D. Floriani, B. Falcidieno, P. Frosini, D. Giorgi, C. Landi, L. Papaleo, M. Spagnuolo, ACM Computing Surveys 40(4), 12:1 (2008)

14. I. Porteous, Geometric Differentiation (Cambridge University Press, 2001)

15. J. Goldfeather, V. Interrante, ACM Trans. Graph. 23(1), 45 (2004)

16. A. Razdan, M. Bae, Comput. Aided Des. 37(14), 1481 (2005)

17. T. Nguyen, I. Debled-Rennesson, in Computer Analysis of Images and Patterns, Lecture Notes in Computer Science, vol. 4673, ed. by W. Kropatsch, M. Kampel, A. Hanbury (Springer Berlin Heidelberg, 2007), pp. 474-481

18. C.L. Bajaj, G. Xu, ACM Trans. Graph. 22(1), 4 (2003)

19. H. Pottmann, J. Wallner, Q.X. Huang, Y.L. Yang, Computer Aided Geometric Design 26(1), 37 (2009)

20. D.J. Tward, J. Ma, M.I. Miller, L. Younes, Int. J. Biomedical Imaging 2013 (2013)

21. D. Coeurjolly, J. Lachaud, J. Levallois, in 17th IAPR International Conference, DGCI 2013. pp. 215-227

22. X. He, N.H.C. Yung, in Pattern Recognition, 2004. ICPR 2004. Proceedings of the 17th International Conference on, vol. 2 (2004), vol. 2, pp. 791-794 Vol.2

23. B. Kerautret, J.O. Lachaud, Pattern Recogn. 42(10), 2265 (2009)

24. T.P. Nguyen, I. Debled-Rennesson, Pattern Recognition 44(1), 32 (2011)

25. K. Raftopoulos, M. Ferecatu, in Computer Vision and Pattern Recognition (CVPR), 2014 IEEE Conference on (2014), pp. 4162-4168

26. S. Manay, D. Cremers, B.W. Hong, A. Yezzi, S. Soatto, Pattern Analysis and Machine Intelligence, IEEE Transactions on 27(11), 1602 (2006)

27. T.B. Sebastian, P.N. Klein, B.B. Kimia, IEEE Trans. Pattern Anal. Mach. Intell. 26(5), $550(2004)$

28. C.D. Manning, P. Raghavan, H. Schutze, Introduction to Information Retrieval (Cambridge University Press, New York, NY, USA, 2008)

\section{APPENDIX I: Proof of Proposition 1}

Proof For the clarity of this proof the dot and double dot will denote the vector first and second derivatives with respect to $s$, whereas the respective scalar derivatives will be denoted by $\frac{d}{d s}$ and $\frac{d^{2}}{d s^{2}}$. For the meanings of $\boldsymbol{r}$ and $\omega$ see section 4 .

For the view function $v_{\xi}\left(s_{*}\right)=\left\|\boldsymbol{\alpha}\left(s_{*}\right)-\boldsymbol{\alpha}(\xi)\right\| \equiv\|\boldsymbol{r}\|$ it is:

$$
\left.\frac{d}{d s} v_{\xi}\left(s_{*}\right) \equiv \frac{d}{d s} v_{\xi}(s)\right|_{s=s_{*}}=\frac{d}{d s}\|\boldsymbol{r}\|=\frac{1}{\|\boldsymbol{r}\|} \boldsymbol{r} \cdot \dot{\boldsymbol{r}}
$$

where is the inner product. Substituting $\boldsymbol{r}=\|\boldsymbol{r}\|(-\sin (\omega), \cos (\omega)), \dot{\boldsymbol{r}}=(1,0)$ we get:

$$
\frac{d}{d s} v_{\xi}\left(s_{*}\right)=-\left.\sin (\omega)\right|_{s=s_{*}}
$$


which is also a proof that the view functions are 1-Lipschitz.

We also have:

$$
\begin{aligned}
\left.\frac{d^{2}}{d s^{2}} v_{\xi}\left(s_{*}\right) \equiv \frac{d^{2}}{d s^{2}} v_{\xi}(s)\right|_{s=s_{*}} & =\frac{d^{2}}{d s^{2}}\|\boldsymbol{r}\|=\left(\frac{1}{\|\boldsymbol{r}\|} \boldsymbol{r}\right) \cdot \dot{\boldsymbol{r}}+\frac{\boldsymbol{r}}{\|\boldsymbol{r}\|} \cdot \ddot{\boldsymbol{r}} \\
& =\left[\boldsymbol{r} \frac{d}{d s}\left(\frac{1}{\|\boldsymbol{r}\|}\right)+\frac{1}{\|\boldsymbol{r}\|} \dot{\boldsymbol{r}}\right] \cdot \dot{\boldsymbol{r}}+\frac{\boldsymbol{r}}{\|\boldsymbol{r}\|} \cdot \ddot{\boldsymbol{r}} \\
& =-\frac{(\boldsymbol{r} \cdot \dot{\boldsymbol{r}})^{2}}{\|\boldsymbol{r}\|^{3}}+\frac{\|\dot{\boldsymbol{r}}\|^{2}}{\|\boldsymbol{r}\|}+\frac{\boldsymbol{r} \cdot \ddot{\boldsymbol{r}}}{\|\boldsymbol{r}\|}
\end{aligned}
$$

and substituting $\boldsymbol{r}=\|\boldsymbol{r}\|(-\sin (\omega), \cos (\omega)), \dot{\boldsymbol{r}}=(1,0), \ddot{\boldsymbol{r}}=-\kappa\left(s_{*}\right)(0,-1)=\left(0, \kappa\left(s_{*}\right)\right)$ we get:

$$
\frac{d^{2}}{d s^{2}} v_{\xi}\left(s_{*}\right)=\left.\kappa\left(s_{*}\right) \cos (\omega)\right|_{s=s_{*}}+\left.\frac{\cos ^{2}(\omega)}{\|\boldsymbol{r}\|}\right|_{s=s_{*}}
$$

and the proof is complete. $\square$

\section{APPENDIX II: Proof of Theorem 1}

For the purpose of the following proofs, we will occasionally consider the view functions as scalars of two parameters, thus we will occasionally use $v(s, \xi)$ meaning $v_{s}(\xi)$, to make it explicit that we work with the two parameters in mind. Both parameters $s$ and $\xi$ are arc lengths measured from the curve's starting point and $v(s, \xi)$ is the plane distance between the corresponding points on the curve. Always the $\operatorname{dot}(\mathrm{s})$ indicate(s) derivative(s) with respect to $s$. Every derivative of $v_{\xi}(s)$ or $v_{s}(\xi)$ and every partial derivative of $v(s, \xi)$ is computed under the assumption that $\xi \neq s$.

Proof (1)

$$
\begin{aligned}
\left.\dot{\phi}_{\alpha}\left(s_{*}\right) \equiv \frac{d \phi_{\alpha}(s)}{d s}\right|_{s=s_{*}} & =\left.\frac{d}{d s} \int_{0}^{\lambda} v_{s}(\xi) d \xi\right|_{s=s_{*}}=\left.\int_{0}^{\lambda} \frac{\partial v}{\partial s}(s, \xi)\right|_{s=s_{*}} d \xi \\
& \left.\stackrel{*}{=} \int_{0}^{\lambda} \frac{\partial v}{\partial s}(\xi, s)\right|_{s=s_{*}} d \xi=\left.\int_{0}^{\lambda} \frac{d v_{\xi}}{d s}(s) d \xi\right|_{s=s_{*}}=\int_{0}^{\lambda} \dot{v}_{\xi}\left(s_{*}\right) d \xi
\end{aligned}
$$

where we have used the Leibniz integral rule. The step of the derivation, marked with * above, is valid due to the fact that $v(s, \xi)=v(\xi, s), \forall s, \xi \in(0, \lambda]$. The integrals make sense since the set where $v_{\xi}(s)$ (or $v_{s}(\xi)$ ) is not differentiable has vanishing measure in $\mathbb{R}$. From Proposition 1, the result in (29) is independent of the variable $\xi$, given the variable $s$ is fixed at $s_{*}$, at the corresponding point of which the Frenet frame is considered (see section 4). This fact permits valid integration over $\xi$, thus:

$$
\dot{\phi}_{\alpha}\left(s_{*}\right)=-\left.\int_{0}^{\lambda} \sin (\omega) d \xi\right|_{s=s_{*}}
$$

which is also a proof that the VAR is $\lambda$-Lipschitz.

Angle $\omega$ is a function of $\xi$ (given $s_{*}$ ) and the integral on the right hand side of (33) quantifies a notion of displacement of the curve with respect to the normal to the curve at $s_{*}$. To see this, consider a point $\boldsymbol{\alpha}(\xi)$ and notice that as $\xi$ traverses the domain of $\boldsymbol{\alpha}$, angle $\omega$ measures the angular displacement of this point with respect to the normal at $s_{*}, \dot{\phi}_{\alpha}\left(s_{*}\right)$ can thus be thought of measuring the total angular displacement of the whole curve with respect to the normal at $s_{*}$. 
Proof (2),(3)

From (33) we get:

$$
\begin{aligned}
\left.\ddot{\phi}_{\alpha}\left(s_{*}\right) \equiv \frac{d^{2} \phi_{\alpha}(s)}{d s^{2}}\right|_{s=s_{*}} & =-\left.\frac{d}{d s}\left(\int_{0}^{\lambda} \sin (\omega) d \xi\right)\right|_{s=s_{*}} \\
& =-\left.\int_{0}^{\lambda} \frac{\partial}{\partial s} \sin (\omega) d \xi\right|_{s=s_{*}} \\
& =-\left.\int_{0}^{\lambda} \cos (\omega) \dot{\omega} d \xi\right|_{s=s_{*}}
\end{aligned}
$$

From the combination of (29) and (31) we get:

$$
-\frac{\partial}{\partial s} \sin (\omega)=-\cos (\omega) \dot{\omega}=\cos (\omega)\left(\kappa(s)+\frac{\cos (\omega)}{\|\boldsymbol{r}\|}\right)
$$

and substituting in (34):

$$
\ddot{\phi}_{\alpha}\left(s_{*}\right)=\kappa\left(s_{*}\right)\left[\left.\int_{0}^{\lambda} \cos (\omega) d \xi\right|_{s=s_{*}}\right]+\left.\int_{0}^{\lambda} \frac{\cos ^{2}(\omega)}{\|\boldsymbol{r}\|} d \xi\right|_{s=s_{*}}
$$

If $s_{*}$ is a local maximum point for $\phi_{\alpha}(s)$, then there exists a neighborhood $U$ of $s_{*}$ such that $\phi_{\alpha}\left(s_{*}\right) \geq \phi_{\alpha}(s)$ for every $s \in U$. In such a case $\ddot{\phi}_{\alpha}\left(s_{*}\right) \leq 0$ and since $\int_{0}^{\lambda} \frac{\cos ^{2}(\omega)}{\|\boldsymbol{r}\|} d \xi>0$ from (36) it follows that:

$$
\kappa\left(s_{*}\right)\left[\left.\int_{0}^{\lambda} \cos (\omega) d \xi\right|_{s=s_{*}}\right]<0
$$

therefore, $\kappa\left(s_{*}\right) \neq 0,\left.\int_{0}^{\lambda} \cos (\omega) d \xi\right|_{s=s_{*}} \neq 0$.

From (36), by setting $A(s)=\int_{0}^{\lambda} \cos (\omega) d \xi$ and $B(s)=\int_{0}^{\lambda} \frac{\cos ^{2}(\omega)}{\|r\|} d \xi$ and given that $\kappa\left(s_{*}\right) \neq 0$, we finally have:

$$
\ddot{\phi}_{\alpha}\left(s_{*}\right)=\kappa\left(s_{*}\right) A\left(s_{*}\right)+B\left(s_{*}\right)\left(\text { i.e., } \kappa\left(s_{*}\right)=\frac{\ddot{\varphi}_{\alpha}\left(s_{*}\right)-B\left(s_{*}\right)}{A\left(s_{*}\right)}\right)
$$

and the proof is complete. $\square$ 